\title{
Impaired Magnesium Protoporphyrin IX Methyltransferase (ChIM) Impedes Chlorophyll Synthesis and Plant Growth in Rice
}

\section{OPEN ACCESS}

Edited by:

Paula Casati,

Consejo Nacional de Investigaciones Científicas y Técnicas (CONICET),

Argentina

Reviewed by:

Peisong Hu,

China National Rice Research Institute

(CAAS), China

Ruidang Quan,

Chinese Academy of Agricultural

Sciences, China

*Correspondence:

Yangsheng LI

lysh2001@whu.edu.cn

Specialty section

This article was submitted to Plant Genetics and Genomics,

a section of the journal

Frontiers in Plant Science

Received: 30 June 2017 Accepted: 14 September 2017 Published: 28 September 2017

Citation:

Wang Z, Hong $X$, Hu K, Wang Y,

Wang $X$, Du S, Li Y, Hu D, Cheng $K$,

An B and Li Y (2017) Impaired

Magnesium Protoporphyrin IX

Methyltransferase (ChIM) Impedes

Chlorophyll Synthesis and Plant

Growth in Rice.

Front. Plant Sci. 8:1694.

doi: 10.3389/fp/s.2017.01694

\begin{abstract}
Zhaohai Wang ${ }^{1,2}$, Xiao Hong ${ }^{1}$, Keke Hu ${ }^{1}$, Ya Wang ${ }^{1}$, Xiaoxin Wang ${ }^{1}$, Shiyun $\mathrm{Du}^{3}$, Yang $\mathrm{Li}^{1}$, Dandan $\mathrm{Hu}^{1}$, Kexin Cheng ${ }^{1}$, Baoguang $\mathrm{An}^{1}$ and Yangsheng $\mathrm{Li}^{1 *}$

'State Key Laboratory of Hybrid Rice, Key Laboratory for Research and Utilization of Heterosis in Indica Rice, Ministry of Agriculture, The Yangtze River Valley Hybrid Rice Collaboration Innovation Center, College of Life Sciences, Wuhan University, Wuhan, China, ${ }^{2}$ The Key Laboratory of Crop Physiology, Ecology and Genetic Breeding, Ministry of Education, School of Agricultural Sciences, Jiangxi Agricultural University, Nanchang, China, ${ }^{3}$ Rice Research Institute, Anhui Academy of Agricultural Sciences, Hefei, China
\end{abstract}

Magnesium protoporphyrin IX methyltransferase (ChIM) catalyzes the formation of magnesium protoporphyrin IX monomethylester (MgPME) from magnesium protoporphyrin IX (MgP) in the chlorophyll synthesis pathway. However, no ChIM gene has yet been identified and studied in monocotyledonous plants. In this study, a spontaneous mutant, yellow-green leaf 18 (yg/18), was isolated from rice (Oryza sativa). This mutant showed yellow-green leaves, decreased chlorophyll level, and climate-dependent growth differences. Map-based cloning of this mutant identified the YGL18 gene LOC_Os06g04150. YGL18 is expressed in green tissues, especially in leaf organs, where it functions in chloroplasts. YGL18 showed an amino-acid sequence similarity to that of ChIM from different photosynthetic organisms. In vitro enzymatic assays demonstrated that YGL18 performed ChIM enzymatic activity, but ygl18 had nearly lost all ChIM activity. Correspondingly, the substrate MgP was largely accumulated while the product MgPME was reduced in yg/18 leaves. YGL18 is required for lightdependent and photoperiod-regulated chlorophyll synthesis. The retarded growth of yg/18 mutant plants was caused by the high light intensity. Moreover, the higher light intensity and longer exposure in high light intensity even made the $y$ g/18 plants be more susceptible to death. Based on these results, it is suggested that YGL18 plays essential roles in light-related chlorophyll synthesis and light intensity-involved plant growth.

Keywords: chlorophyll synthesis, light intensity, magnesium protoporphyrin IX methyltransferase (ChIM), photoperiod, plant growth, rice (Oryza sativa), yellow-green leaf 18 (ygl18)

\section{INTRODUCTION}

Chlorophyll is the main component of the photosynthetic pigments found in plants, algae and cyanobacteria (Czarnecki and Grimm, 2012). Chlorophyll is extremely important in photosynthesis because of its essential roles in harvesting light energy to form chemical energy (Grossman et al., 1995; Fromme et al., 2003).

Chlorophyll synthesis from glutamyl-tRNA to chlorophyll $b$ requires a 15-step of enzymatic reaction, and all 27 genes encoding these 15 enzymes have been identified in higher plants 
represented by Arabidopsis (Arabidopsis thaliana) (Beale, 2005; Nagata et al., 2005). However, only 10 genes encoding seven enzymes in the chlorophyll synthesis pathway have been identified in rice (Oryza sativa). Coproporphyrinogen III oxidase converts coproporphyrinogen III to protoporphyrinogen IX. RLIN1, encoding a putative coproporphyrinogen III oxidase in rice, was cloned from a lesion mimic mutant (Sun et al., 2011). Magnesium chelatase consists of three subunits (ChlH, ChlI, and ChlD) and it catalyzes the formation of $\mathrm{Mg}$-protoporphyrin IX from protoporphyrin IX. OsChlH was identified via a T-DNA insertional mutant that had the chlorina and lethal phenotype (Jung et al., 2003). The OsChlD and OsChlI genes were cloned from the spontaneous chlorina mutants, chl1 and chl9, respectively (Zhang et al., 2006). OsChlD was also cloned with the spontaneous yellow-green leaf mutant $y g l 7$, and the RNAi plants of OsChlD were found to have a lethal phenotype (Deng et al., 2014). OsCRD1 encodes MgProtoporphyrin IX monomethyl ester cyclase, transforming magnesium protoporphyrin IX monomethylester (MgPME) into divinyl protochlorophyllide. OsCRD1 was cloned by using a pale-green leaf mutant $m 167$ and a yellow leaf mutant $y l-1$, and found to have a dual role in chlorophyll synthesis and photosynthesis capacity (Sheng et al., 2017; Wang et al., 2017). NADPH:protochlorophyllide oxidoreductase catalyzes the photoreduction of protochlorophyllide to chlorophyllide. $O S P O R B$, encoding the NADPH:protochlorophyllide oxidoreductase $\mathrm{B}$, was cloned by using the faded green leaf mutant $f g l$, and it was found essential for chlorophyll synthesis under high light conditions (Sakuraba et al., 2013). OsDVR encodes the divinyl reductase, which catalyzes the conversion of divinyl chlorophyll(ide) $a$ to monovinyl chlorophyll(ide) $a$; OsDVR was cloned by using the spontaneous yellow-green leaf mutant 824ys (Wang et al., 2010). Chlorophyll synthase catalyzes the esterification of chlorophyllide to form chlorophyll $a$, and YGL1, encoding the chlorophyll synthase, has been cloned with the chlorophyll-deficient mutant $y g l 1$ (Wu et al., 2007). Chlorophyll $b$ is synthesized from chlorophyll $a$ by chlorophyll $a$ oxygenase; both OsCAO1 and OsCAO2 genes were identified to encode the chlorophyll $a$ oxygenase; the Tos 17 insertion mutant of OsCAO1 showed a pale green leaf phenotype, but the insertion mutant of OsCAO2 was similar to the wild type (Lee et al., 2005). OsCAO1 was also identified through the pale green leaf mutant $p g l$, impacting leaf senescence and thus indirectly affecting grain yield and quality (Yang et al., 2016).

Magnesium protoporphyrin IX methyltransferase (ChlM) is another key enzyme in the chlorophyll synthesis pathway. The ChlM gene has been identified and studied in several species. Through cDNA cloning and protein expression, the enzymatic kinetics of the ChlM in Synechocystis sp. PCC6803 have been elaborately studied: it catalyzes the methyl transfer from the

\footnotetext{
Abbreviations: CDS, coding sequence; ChlM, magnesium protoporphyrin IX methyltransferase; DIS, days in sunlight; FW, fresh weigh; GST, glutathione$S$-transferase; MgP, magnesium protoporphyrin IX; MgPME, magnesium protoporphyrin IX monomethylester; qRT-PCR, quantitative real-time reverse transcription polymerase chain reaction; SAM, S-adenosylmethionine; yglls, yellow-green leaf 18; YFP, yellow fluorescent protein.
}

common methyl donor $S$-adenosylmethionine (SAM) to the carboxyl group of the $\mathrm{C} 13$ propionate side chain of magnesium protoporphyrin IX $(\mathrm{MgP})$, enabling the formation of $\mathrm{MgPME}$ and S-adenosylhomocysteine (Shepherd et al., 2003; Shepherd and Hunter, 2004). The structure of Synechocystis ChlM has also been analyzed to illustrate its catalytic mechanism (Chen et al., 2014). Two Chlamydomonas reinhardtii mutants defective in ChlM were identified; both were yellow in the dark and dim light, and their growth was inhibited under higher light intensities (Meinecke et al., 2010). In tobacco (Nicotiana tabacum), ChlM cDNA was cloned to study its enzymatic function, and ChlM was found to interact with the $\mathrm{ChlH}$ subunit of $\mathrm{Mg}$ chelatase (Alawady et al., 2005). Inhibited and excessive expression of tobacco ChlM revealed its functions involved in the inverse activation of protoheme and $\mathrm{Mg}$ porphyrin synthesis (Alawady and Grimm, 2005). In Arabidopsis, the ChlM gene was identified by cDNA cloning and the ChlM enzymatic activity was verified in vitro (Block et al., 2002). In addition, an Arabidopsis knockout mutant of the ChlM gene showed that this gene is essential to form chlorophyll and subsequently for the formation of photosystem complexes (Pontier et al., 2007). Recently, it was proposed that ChlM was regulated by the NADPH-dependent thioredoxin reductase $\mathrm{C}$ on the redox status of conserved cysteine residues of ChlM (Richter et al., 2013). Further research has showed that three conserved cysteines are essential for the activity and redox regulation of Arabidopsis ChlM (Richter et al., 2016). A study of the pea plant (Pisum sativum) revealed that the ChlM activity is dependent on its folate status (Van Wilder et al., 2009). However, no ChlM gene has yet been identified and studied in monocotyledonous plants.

This study performed a map-based cloning of the yellow-green leaf 18 (ygl18) locus in rice. Doing so revealed that ygl18 harbored a single-base substitution in the OsChlM gene, resulting in a single amino acid change in the protein. The ChlM activity of YGL18 was checked, while ygl18 was attenuated on the enzymatic function, leading to the accumulation of $\mathrm{MgP}$ and the reduction of MgPME in the ygl18 mutant. On the basis of the phenotypic and molecular characterization of $y g l 18, Y G L 18$ is proposed to play multiple yet important roles in chlorophyll synthesis and plant growth.

\section{MATERIALS AND METHODS \\ Plant Materials and Growth Conditions}

The ygl18 mutant was isolated from the rice cultivar "Guangzhan63S" (Oryza sativa L. subsp. indica), and its yellowgreen leaf phenotype was stably inherited. For our experiments, the plants were grown in the paddy field, green house, or plant growth chambers. For the experimental greening of the etiolated seedlings, wild-type and $y g l 18$ seeds were germinated and then grown in the chamber $\left(30^{\circ} \mathrm{C}, 24 \mathrm{~h}\right.$ dark) for 6 days, before exposure to light $\left(150 \mu \mathrm{mol} \mathrm{m} \mathrm{m}^{-2} \mathrm{~s}^{-1}\right)$. For expression patterns of YGL18 in different tissues, wild-type and ygl18 plants were grown in the chamber $\left(150 \mu \mathrm{mol} \mathrm{m} \mathrm{m}^{-2} \mathrm{~s}^{-1}\right.$ light/dark, $10 / 14 \mathrm{~h}$, $26 / 22^{\circ} \mathrm{C}$ ). For daily expression experiments, wild-type and ygl18 seedlings were cultured in the chamber $\left(28^{\circ} \mathrm{C}\right)$ for 12 days under 
short-day ( $8 \mathrm{~h}$ light, $150 \mu \mathrm{mol} \mathrm{m}^{-2} \mathrm{~s}^{-1}$ ) or long day ( $14 \mathrm{~h}$ light, $150 \mu \mathrm{mol} \mathrm{m}^{-2} \mathrm{~s}^{-1}$ ) conditions before sampling.

\section{Analysis of Pigments}

Chlorophyll was extracted from the plant leaves with ice-cold $80 \%$ acetone, and the chlorophyll contents per gram of leaf fresh weigh (FW) were determined as described previously (Lichtenthaler, 1987).

\section{Map-Based Cloning}

To construct the genetic population, the rice cultivars "Nipponbare" (Oryza sativa L. subsp.japonica), "02428" (Oryza sativa L. subsp. japonica), and "9311" (Oryza sativa L. subsp.indica) were used in this study.

The $F_{2}$ population from the cross between ygl18 and "Nipponbare" were used for the preliminary mapping of the YGL18 locus. Ninety-two $F_{2}$ mutant plants were detected via simple sequence repeat markers, which were well distributed across all 12 chromosomes, thus allowing ygl18 and "Nipponbare" to be distinguished. Five new markers (Y11, Y13, Y15, Y20, and Y37) were successfully developed to distinguish ygl18 and "9311" based on the DNA sequence differences between the indica and japonica rice varieties. Therefore, the $\mathrm{F}_{2}$ population from the cross between ygl18 and "9311" were used for the fine mapping, for which a total of $910 \mathrm{~F}_{2}$ mutant plants were tested. Primer sequences of the markers were listed in Table S1.

\section{Complementation of YGL18}

To test the C-to-T mutation, the PCR amplification products were sequenced with the primer pair YGL18 Mutation (Table S2). For complementation of the ygl18 phenotype, two DNA fragments covering the 2,732-bp upstream promoter, the 981bp gene region and the 1,433-bp downstream terminator of the gene LOC_Os06g04150, were firstly amplified from the genomic DNA of wild-type Guangzhan63S by using the primers YGL18C1 and YGL18C2 (Table S2). Then, the amplified products were constructed into the binary vector pBWA(V)HII (reconstructed from pCAMBIA1301), by using the Aar I restriction site on YGL18C1 and YGL18C2, and the $B s a$ I restriction site on pBWA(V)HII for the digestionlink reactions. The resultant seamlessly cloned 5,146-bp DNA fragment was sequenced to confirm its identity with the sequence found in the wild-type Guangzhan63S. This vector constructed for functional complementation was termed pYGL18C, while the empty pBWA(V)HII was renamed pEmvC. Both vectors pYGL18C and $\mathrm{pEmvC}$ were transformed into the calli of $y g l 18$ mutant through the Agrobacterium (EHA105)-mediated method. Positive transgenic plants were identified by using $H Y G$ primer pairs (Table S2) specific for the amplification of the hygromycin gene.

\section{Bioinformatic Analysis}

To reveal the specific ChlM encoded by YGL18, BLASTP searches were conducted with the full-length amino acid sequence on NCBI (http://www.ncbi.nlm.nih.gov/). The YGL18 gene information was further investigated on Rice Genome
Annotation Project website (http://rice.plantbiology.msu.edu/). Full-length amino acid sequences were used for multiple sequence alignments performed with the ClustalX2 software. A consensus phylogenetic tree was built with the MEGA5 software by using the neighbor-joining method with 1,000 random bootstrap replicates.

The online software ChloroP 1.1 Server (http://www.cbs.dtu. $\mathrm{dk} /$ services/ChloroP/) and TargetP 1.1 Server (http://www.cbs. $\mathrm{dtu} . \mathrm{dk} /$ services/TargetP/) were used to predict the subcellular localization of YGL18.

\section{Gene Expression Analysis}

The collected samples were immediately frozen in liquid nitrogen, and then stored at $-80^{\circ} \mathrm{C}$ before use. Total RNAs of the various tissues were isolated with the TRIzol reagent (Invitrogen). After the DNase treatment, about $5 \mu \mathrm{g}$ of RNAs were used for the cDNAs synthesis that utilized the M-MLV reverse transcriptase (Promega) in a 50- $\mu \mathrm{l}$ reaction mixture. Quantitative real-time reverse transcription polymerase chain reaction (qRT-PCR) was performed with the $2 \times$ SYBR Green Master Mix reagent (Bio-Rad) in a 96-well plate of the Bio-Rad CFX96 real time PCR system. Eight rice reference genes: TI (LOC_Os01g05490), ARF (LOC_Os0 5g41060), EF-1 $\alpha$ (LOC_Os03g08020), UBC (LOC_Os02g42314), Profilin-2 (LOC_Os06g05880), Edf (LOC_Os08g27850), PtfS (LOC_Os07g34589), and Actin1 (LOC_Os03g50885), were used to find the stable internal standards by geNorm as described (Vandesompele et al., 2002; Wang et al., 2016). The selected internal standards for the different experimental conditions were showed in Figure S1. All primers used for qRT-PCR analysis are listed in Table S3, with good PCR efficiencies (80-100\%) detected by using ten-times diluted gradients of the total cDNAs.

\section{Subcellular Localization of YGL18}

To fuse YGL18 with the yellow fluorescent protein (YFP), the coding sequence (CDS) of YGL18 without the termination codon was amplified by using the primer pair YGL18-YFP (Bbs I restriction site) (Table S2), and then cloned into the vector pBWD(LB)-p35SYFP (Bsa I restriction site). The fusion constructs (p35s::YGL18-YFP) and the control (p35S::YFP) were then transformed into the rice protoplasts as previously described (Yu et al., 2014). The samples were subsequently examined with an FV1000 confocal system (OLYMPUS FLUOVIEW).

\section{Recombinant Protein Construction, Expression and Purification}

To generate the glutathione-S-transferase (GST) fusion constructs, GST-YGL18 and GST-ygl18, 981-bp full-length coding sequences of YGL18 and ygl18 were amplified, respectively, by using the primer pair GST-YGL18/ygl18 (Table S2), and then inserted into pGEX-6P-1 by using the restriction enzyme sites BamH I and EcoR I. The subsequent recombinant protein construction, expression and purification were performed as described previously (Wang et al., 2015). 


\section{ChIM Enzymatic Assay}

The enzymatic reactions were performed as described previously (Richter et al., 2013) with some modifications. The reaction mixture included $50 \mathrm{mM}$ Tris- $\mathrm{HCl}$ (pH7.5), $500 \mu \mathrm{M}$ NADPH, $500 \mu \mathrm{M}$ SAM, and $2 \mu \mathrm{M} \mathrm{MgP}$ in a final volume of $100 \mu \mathrm{l}$. After the addition of the recombinant proteins (GST, GST-YGL18, or GST-ygl18), the reactions were incubated at $30^{\circ} \mathrm{C}$ for $1 \mathrm{~h}$. These reactions were stopped by adding $500 \mu \mathrm{l}$ of acetone: $\mathrm{NH}_{4} \mathrm{OH}$ (9:1), and the product formation was determined by HPLC as described below.

\section{Extraction of MgP and MgPME from Plant Leaves}

Plant leaves were sampled and frozen in liquid nitrogen, and then stored at $-80^{\circ} \mathrm{C}$ before use. The harvested leaves $(100 \mathrm{mg})$ were powdered in liquid nitrogen with a pestle and mortal. The MgP and MgPME contents were extracted by using chilled acetone according to the methods as previously described (Mochizuki et al., 2008).

\section{Quantification of MgP and MgPME by HPLC}

The HPLC analysis of MgP and MgPME was conducted as previously described (Mochizuki et al., 2008) with some modifications. Briefly, here we used a ZORBAX SB-C18 column $(5 \mu \mathrm{m}, 4.6 \mathrm{~mm} \times 250 \mathrm{~mm})$, eluting with a linear gradient of solvent $\mathrm{B}$ ( $85 \%$ methanol, $15 \% 0.1 \mathrm{M}$ ammonium acetate, $\mathrm{pH} 5.2$ ) in solvent $\mathrm{A}\left(55 \% \mathrm{H}_{2} \mathrm{O}, 30 \%\right.$ methanol, $15 \% 0.1 \mathrm{M}$ ammonium acetate, pH5.2) as follows: 0 to $100 \%$ solvent B over $7 \mathrm{~min}$ and $100 \%$ solvent $\mathrm{B}$ for $16 \mathrm{~min}$, with a flow rate of $1 \mathrm{~mL} \mathrm{~min} \mathrm{~m}^{-1}$. To identify MgP and MgPME in samples, standard curves were constructed with the authentic standards for each independent experiment, as shown in Figure S2 (for in vitro ChlM enzymatic experiment) and Figure S3 (for content measurement in leaves). $\mathrm{MgP}$ was purchased from Frontier Science, and MgPME was chemically synthesized by treating $\mathrm{MgP}$ with diazomethane in methanol as previously described (Strand et al., 2003).

\section{RESULTS}

\section{The Phenotype of $y g / 18$}

The spontaneous $y g l 18$ mutant was isolated from the rice cultivar "Guangzhan63S" (Oryza sativa subsp. indica) during its reproduction. In the paddy field, the yglls mutant plants could be easily distinguished by their yellow-green leaves at the seedling stage (Figure 1A). Upon examination, the chlorophyll content was greatly decreased in ygl18 leaves when compared with that of the wild type (Figure 1B). But the chlorophyll $a / b$ ratio appeared higher in the yglis mutant (Figure 1C). When the plants were cultivated in a natural field in Wuhan $\left(113^{\circ} 41^{\prime} \mathrm{E}\right.$ $115^{\circ} 05^{\prime} \mathrm{E}, 29^{\circ} 58^{\prime} \mathrm{N}-31^{\circ} 22^{\prime} \mathrm{N}$; Hubei province) from June to September, the $y g l 18$ mutants showed severely retarded growth (Figure 1D); sometimes the ygl18 mutants could not even grow and just withered away. However, the growth status of the $y g l 18$ mutants was nearly similar to that of the wild type (Figure 1E), when the plants were cultivated in a natural field in Lingshui $\left(109^{\circ} 45^{\prime} \mathrm{E}-110^{\circ} 08^{\prime} \mathrm{E}, \quad 18^{\circ} 22^{\prime} \mathrm{N}-18^{\circ} 47^{\prime} \mathrm{N}\right.$; Hainan province) from December to March. Therefore, the ygl18 phenotypic characterization implied that the YGL18 is probably involved in chlorophyll synthesis and climate-dependent plant growth in rice.

\section{Map-Based Cloning of YGL18}

For the genetic analysis, four hybrid populations were constructed from the crosses between the ygl18 mutant and "Nipponbare," "02428," and "9311." All $F_{1}$ plants from these crosses displayed the wild-type green leaves, while their $\mathrm{F}_{2}$ progenies all showed a segregation ratio of 3:1 (green: yellowgreen plants, $\chi^{2}<\chi_{0.05}^{2}=3.84 ; P>0.05$; Table S4). These results suggested that the yellow-green leaf phenotype of the ygl18 mutant is controlled by a single recessive nuclear gene.

Ninety-two yellow-green plants, coming from the $F_{2}$ cross population between the ygl18 mutant and "Nipponbare," were used for the preliminary mapping of the YGL18 locus. The YGL18 locus was mapped between the SSR makers chr6mm0129 and chr6mm0287 on chromosome 6 (Figure 2A), and no polymorphism marker between ygl18 and "Nipponbare" was found in this region. However, five new markers (Y11, Y13, Y15, Y20, and Y37) in or beside the preliminary region were developed, showing polymorphism between ygl18 and "9311." Accordingly, by using $910 \mathrm{~F}_{2}$ yellow-green plants generated from the cross of $y g l 18$ and "9311," the YGL18 locus was additionally mapped between the new makers Y37 and Y11 (Figure 2B). As a result, the YGL18 gene was eventually limited in a $343-\mathrm{kb}$ region between markers chr6mm0129 and Y11 (Figure 2B). A total of 41 putative genes were predicted according to the NCBI website. Genomic sequences of the $y g l 18$ mutant were sequenced for all these 41 genes, and one point mutation was found on the CDS region of the 21st gene, LOC_Os06g04150 (Figure 2C). The single nucleotide substitution of cytosine (C) to thymine (T) occurred at the 673-bp position of the CDS, resulting in a single amino acid change from leucine (Leu) to phenylalanine (Phe). This C-to-T mutation was existed for ygl18 only. It did not occur in nine different normal rice cultivars, and similarly, all the tested $\mathrm{F}_{2}$ yellow-green plants had $\mathrm{T}$ at this nucleotide site, while the tested $\mathrm{F}_{2}$ green plants owned the $\mathrm{C}$ or $\mathrm{C} / \mathrm{T}$ (Table S5). These results suggested that LOC_Os06g04150 is the likely YGL18 candidate.

A functional complementation experiment was conducted to confirm that the mutation in LOC_Os06g04150 was responsible for the ygl18 phenotype. A 5146-bp genomic fragment of LOC_Os06g04150 from the wild-type Guangzhan63S was constructed into the plasmid pYGL18C and then transferred into the $y g l 18$ calli via an Agrobacterium tumefaciens-mediated transformation, while the empty vector $\mathrm{pEmvC}$ was transformed as the control (Figure 2D). The yellow-green leaves were completely restored to normal color in nine independent transgenic lines with pYGL18C, but they were unchanged in eight independent control transgenic lines with $\mathrm{pEmvC}$ (Figures 2E-G). The chlorophyll contents and the chlorophyll $a / b$ ratio in the complementation lines with pYGL18C were also restored to normal, as they occur in the wild type (Figures $\mathbf{2 H}, \mathbf{I}$ ). Hence, these results confirmed that LOC_Os06g04150 indeed is the YGL18 gene. 


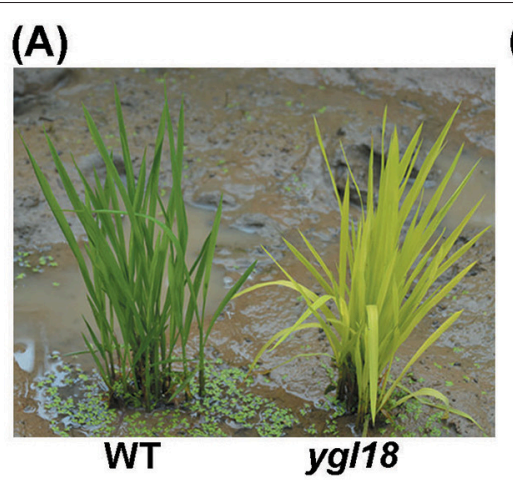

(B) (C)
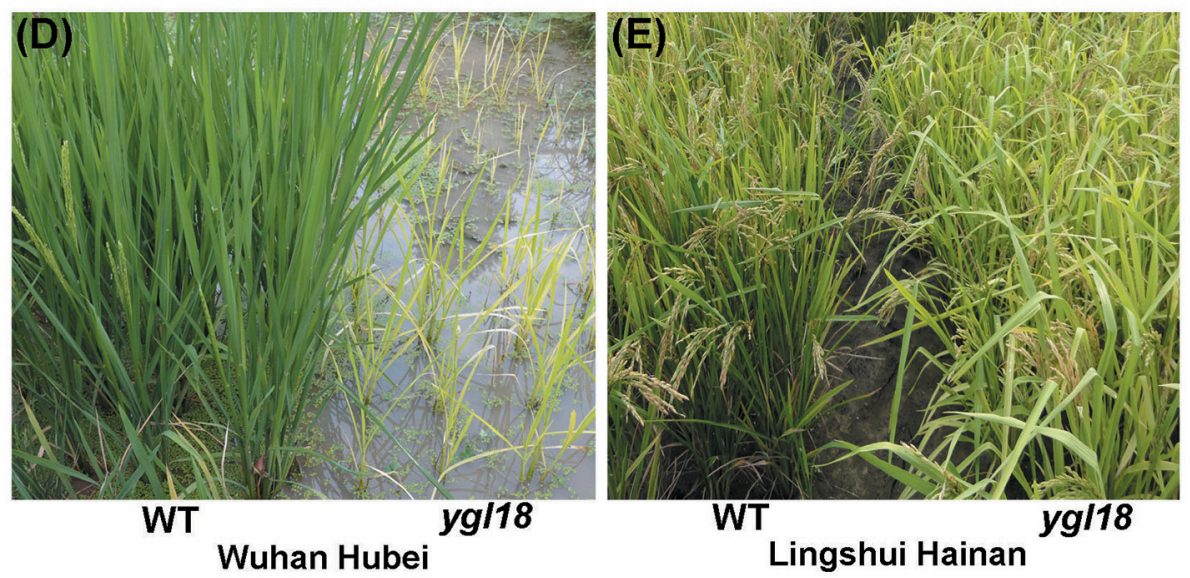

FIGURE 1 | Phenotypic characterization of the wild-type and yg/18 mutant plants. (A) Plants at the seedling stage (about 15 days old) in the paddy field. (B) Chlorophyll contents. FW, fresh weight. (C) Chlorophyll a/b ratio. (B,C) Data represent the mean \pm SD of three biological replicates (Student's $t$-test: ${ }^{\star \star \star} P<$ 0.0005). (D) Plants growing in a natural field in Wuhan of Hubei province in China. (E) Plants growing in a natural field in Lingshui of Hainan province in China.

\section{YGL18 Is Mainly Expressed in Leaves and Localized in Chloroplasts}

The expression patterns of YGL18 in different rice tissues were analyzed by qRT-PCR. The YGL18 mRNAs were most abundant in the leaf, moderate in the stem and panicle, with very few occurring in the root (Figure 3A). We further investigated the expression of YGL18 in the top leaf of plants at different developmental time points. Expression levels of YGL18 steadily increased in the leaves of 15-, 45-, 90-, and 130- day-old wild-type plants, and these levels were even higher in the leaves of $y g l 18$ plants compared with those of the wild type (Figure 3B). Expression levels of YGL18 were also tested in the first, second, and third plant leaves from the top. While they were approximately equal among wild-type leaves, they were, respectively higher in the $y g l 18$ leaves when compared with those of the wild type (Figure $3 \mathrm{C}$ ). These results suggested that YGL18 mainly operates in the green tissues, especially in leaf organs, and that YGL18 is upregulated in the $y g l 18$ mutant leaves.

To reveal where the YGL18 protein functions in the cells, the online software ChloroP 1.1 Server and TargetP 1.1 Server were firstly used to predict the subcellular localization of YGL18. The YGL18 protein was found to contain chloroplast transit peptides and so it should target into the chloroplasts. To confirm this prediction, YGL18 was fused with YFP. The 35S::YGL18-YFP and 35S::YFP were separately introduced into the rice protoplasts. The YFP fluorescence signal was found to co-localize with the area of chloroplast autofluorescence in those protoplasts transformed with 35S::YGL18-YFP (Figures 4A-D). By contrast, the YFP fluorescence signal appeared throughout the cytoplasm, but not in the chloroplasts, when protoplasts were transformed with 35S::YFP (Figures 4E-H). These results indicated that the YGL18 protein is localized in the chloroplasts where it presumably performs its function.

\section{YGL18 Encodes a Putative ChIM, and a Conserved Amino Acid Is Substituted in the ygl18 Protein}

There is only one copy of the YGL18 gene in the rice genome as detected by NCBI blastn search. Specifically, the 981-bp CDS encoding a protein of 326 amino acids is predicted on Rice Genome Annotation Project website. The NCBI blastp revealed that YGL18 is the ChlM gene, encoding a putative ChlM. Alignment of the YGL18 and ygl18 proteins with their homologs from nine different photosynthetic organisms was also performed. The result showed that many of the amino acid residues-in the region from 95 to 319 in the 


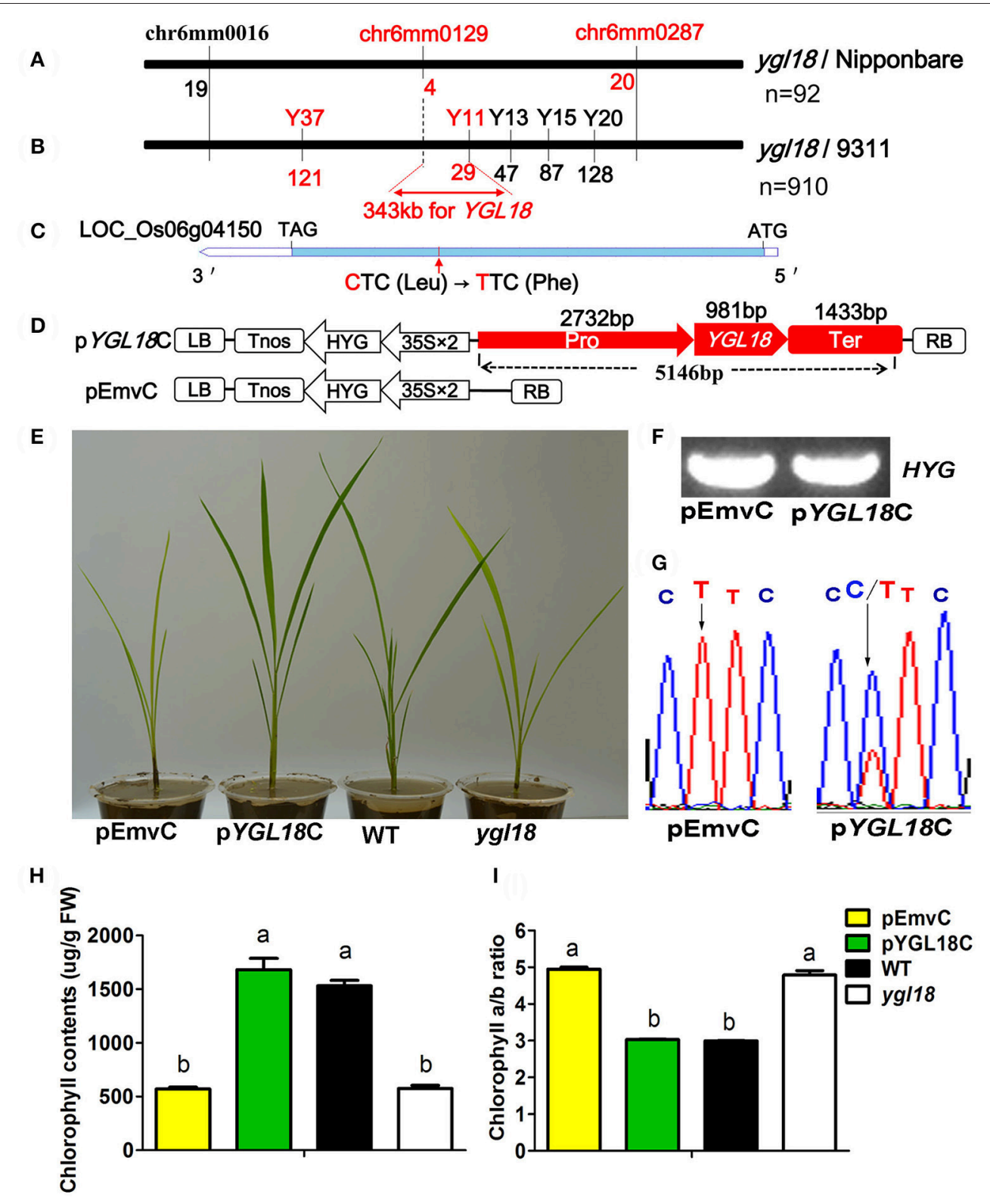

FIGURE 2 | Map-based cloning and functional complementation of YGL18. (A) Preliminary mapping identified the YGL18 locus as occurring between chr6mm0129 and chr6mm0287 on the rice chromosome 6 , by using $92 \mathrm{~F}_{2}$ recessive individuals from the cross of $y g / 18$ and "Nipponbare." The number of recombinants is indicated below the marker. (B) The YGL18 locus was mapped to lie between markers $Y 37$ and $Y 11$ by $910 F_{2}$ recessive individuals from the cross of $y g / 18$ and "9311." Accordingly, a candidate region of $343 \mathrm{~kb}$ between chr6mm0129 and Y11 was summarized. (C) Gene structure of the YGL18 candidate, LOC_Os06g04150. The blue bar indicates the CDS region. The red arrow indicates the point mutation of C-T, leading to the amino acid exchange of Leu to Phe. (D) Vectors for the complementary experiment. The functional complementary vector pYGL18C contains the promoter (Pro), gene region (YGL 18), and terminator (Ter) of LOC_Os06g04150; pEmvC is the empty vector control. LB, left border; Tnos, the nopaline synthase terminator; HYG, the hygromycin gene; 35S, cauliflower mosaic virus $35 \mathrm{~S}$ promoter; RB, right border. (E) Transgenic plants of $\mathrm{pEmvC}$ and $\mathrm{pYGL} 18 \mathrm{C}$ grown in the green house. (F) Positive amplification of the transgenic marker element (HYG gene) in the transgenic plants of pEmvC and pYGL18C. (G) Sequence analysis of the C-to-T mutation site in $T_{0}$ transgenic plants of $\mathrm{pEmvC}$ and pYGL18C. (H) Chlorophyll contents in the transgenic plant leaves of pEmvC and pYGL18C. FW, fresh weight. (I) Chlorophyll a/b ratio in the transgenic plant leaves of pEmvC and pYGL18C. In (H,I) Data represent the mean \pm SD of three biological replicates (one-way ANOVA analysis). Different letters indicate values are statistically different based on a one-way ANOVA analysis.

YGL18 amino acid sequences-were highly conserved among the tested organisms, and the amino acid substitution of Leu (L) to Phe (F) at position 225 in the ygl18 amino acid sequences was originally a fully conserved residue (Figure 5), implying the Leu at this position was biologically important. We then analyzed the possible phylogenetic relationships between YGL18 and its homologs by constructing a bootstrap consensus phylogenetic tree. The rice YGL18 was more closely related 

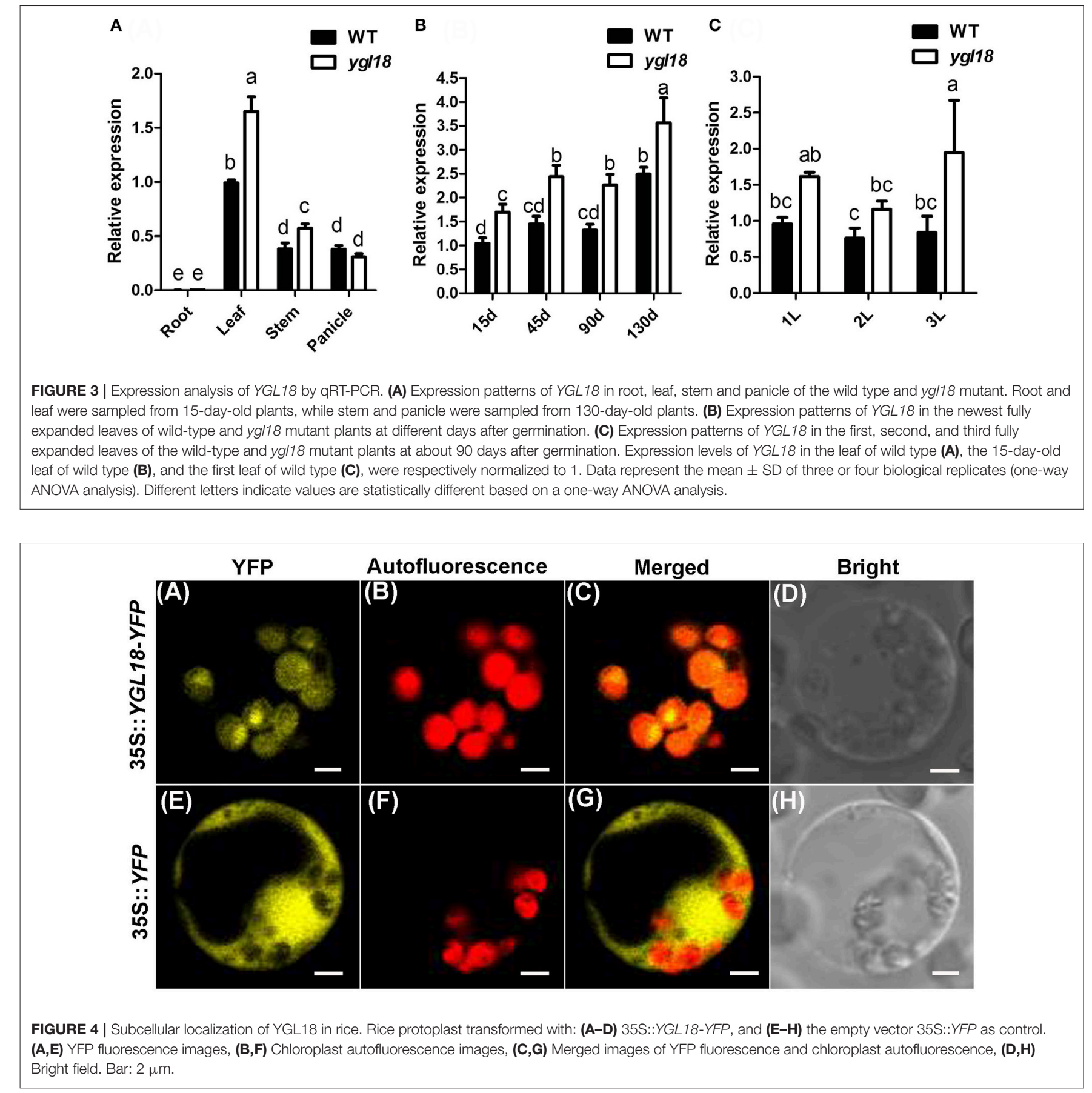

to the ChlM orthologs of monocotyledonous plants Sorghum bicolor and Zea mays than to those of other species (Figure S4). In addition, the YGL18 protein shared over 50\% amino acid identity with all the analyzed ChlM orthologues in different photosynthetic organisms, including the prokaryote Synechocystis sp. PCC6803 (Figure S4). Together, these results suggested that YGL18 encodes a putative and functionally conserved ChlM protein in rice, and that the substitution of the single conserved amino acid in ygl18 protein may impact its function.

\section{YGL18 Performs ChIM Enzymatic Activity While the Function of ygl18 Is Starkly Weakened}

To test the ChlM activity of the YGL18 and ygl18 proteins, recombinant GST-YGL18 and GST-ygl18 proteins were produced. Theoretically, the molecular weights of GST, YGL18, and ygl18 proteins are 26, 34.93, and $34.96 \mathrm{kDa}$, respectively. After induction, GST, GST-YGL18 (about $61 \mathrm{kDa}$ ), and GSTygl18 (about $61 \mathrm{kDa}$ ) were highly expressed at the expected sizes (Figure S5, lanes 2-4). Column-purified proteins were 
YGL18 --MARAAVSTAPLSRVHSPPPLIPRHPHSHSRVGLLHPQ--RKALTTAAALPPAADLPPLSLPAAAA 63 Ygl18 --MARAAVSTAPLSRVHSPPPLIPRHPHSHSRVGLLHPQ--RKALTTAAALPPAADLPPLSLPAAAA 63 SbChIM --MARAGVSTAPLSRLHSLPPRPSLLHNPQPNVLLIRPQQRKAPATTAAAL PTAADLPELSLPAAAA 65 ZMChIM --MARAGVSTAPLSRTHLIPPRPSLLHNTQPHSLLLRPQ-RKAPATTAAALPPAADFPALSIPAAAA 64 NTChIM MAFSS PLF SPVNFALNPNPQ-IHPKCTKPNRRN--ESVCAIPPLSTATDISAVTGPLDGTTLAVLGG 64 VVChIM MALSPTESPPVCFKKNTTESPNTPKFHKPTTKTPTQTLSAIPPISTAADLSAVAVPFDGTTLAVIGG 67 PtChIM MAFTSSIFSHIPLSNQRIFP---PNSHKPKPPRTTFTTAALPPLAAVT-----AADLDGTTLAVIGG 59 GMChIM MAFSSSIWSSSIFVPNPNRTTISTRF SHTPSKPPLSPAFAIPPLSTAT-ATDVSGVIDGTTIAVVSG 66 AtChIM MPFAPSLLSSSSSVSQFLPRFPNATRFNVTPRSRAATVVAASVTDLAG--------VDSTTIAVLGG 59

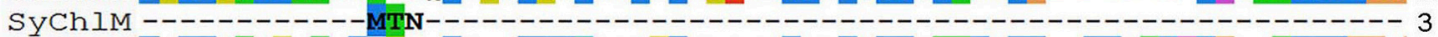
CIChIM MQCLNTKPFTSARPAVARRSVVAHASRPÄQAAALAISAMTVATTSAAMASEIAQTADVGSLTFAVGGV 67

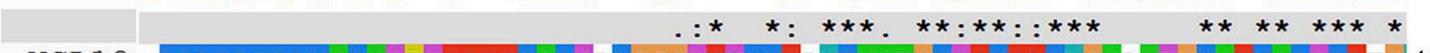

YGL1 8 -AAAALAAAVSLSDPERRRRAQAEAAGGGDKEAVRAYFNSTGFERWRKIYGSATDGVNRVQLDIREG 129 Yg118 -AAAALAAAVSLSD PERRRRAQAEAAGGGDKEAVRAYFNSTGEERWRKIYGSATDGVNRVQLDIREG 129 SbChIM -AAAALAAAVSLSDPERRRRAQAEAAGGGDKEAVRAYFNSTGFERWRKIYGSATEGVNRVQLDIREG 131 ZMChIM -AAAALAAAVSLSDPERRRRSQAEAAGGGDKEAVRAYFNSTGFERWRKIYGSATEGVNRVQLDIREG 130 NtChIM GSVAALAAILSLADPERRRQLQAEEVGGGDKEVVREYFNNNGFQRWKKIYG-DTDDVNKVQLDIRLG 130 VVCh IM GSVAALAAVLSLTDPERRRQLQAEEVGGGDKEVVKEYFNNSGFQRWKKIYG-ETDDVNKVQLDIRLG 133 PtChIM GSVAALAAAISLTDPERRRRLQAEEVGGGDKEVVREYFNNSGFQRWRKIYG-ETDEVNRVQKDIRLG 125 GmChIM GFVAALAAVLSLTDPERRRQMQAEEVGGGDKEVVREYFNNSGFQRWKKIYG-DTDEVNRVQRDIRLG 132 AtCh IM GSVAALAAMVSLTDPERRRKLQAEEVGGGDKEVVREYFNSTGFERWRKIYG-ETDEVNRVQKDIRLG 125 SYChIM--------------------AALDDKTIVRDYFNSTGFDRWRRIYG--DGQVNFVQKDIRVG 43 CrChIM GAVVGLGALLVATDPQKRRSEQMKSFDGDEKEAVKDYFNTAGFERWRKIYG-ETDEVNKVQIDIRTG 133

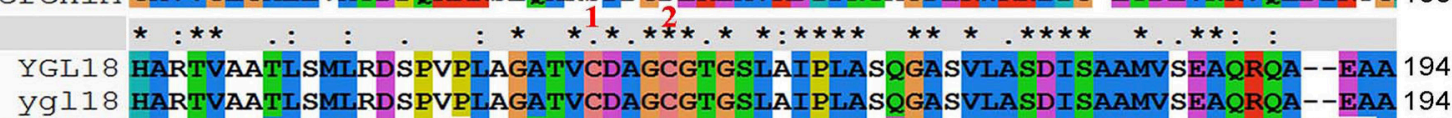
SbChIM HAQTVAAALSMLRDSPLELAGTTVCDAGCGTGSLAIPLAAEGADVLASDISAAMVSEAQRQASLAQA 198 ZmChIM HAQTVAAALAMLRD SPLELAGATVCDAGCGTGSLAIPLAAAGADVLASDISAAMV SEAQRQA----A 193 NTCh IM HSKTVENVMKML TEE-GSLQGVTVCDAGCGTGCL SI PLAKFGAVVSASDISASMVAEAQKQAQEELF 196 VVChIM HSKTVENVMKMLTDE-RSLEGVTVCDAGCGTGCLSIPLAKQGAIVTASDISAAMVAEAEKQAREELL 199 PtChIM HSQTVENVLKMLKDE-GSLEGVSVCDAGCGTGCLAIPLAKEGAAVYASDISAAMVAEAEKQAKEQLQ 191 GMCh IM HAKTVENTISMLKDE-GSLQGITVCDAGCGTGSLSIPLAKEGAVVFASDISAAMVAEAEKQAKEQLV 198 AtChIM HAKTVENTMLML TED-RSLAGVTVCDAGCGTGLISIPLAKEGAIVSASDISAAMVAEAFMKAKAQLP 191 SYChIM HQQTVDSVVAWLVAD-GNLPGLLVCDAGCGVGSI SIPLAQAGALVYGSDISEKMVGEAQQKAQEVLA 109 CrChIM HAQTVDKVLRWVDEE-GSVQGITVADCGCGTGSIAIPLALRGAAVSASDISAAMA SEAEQRYQQAVA 199

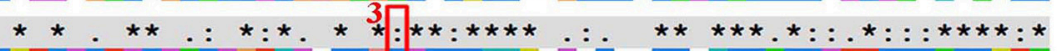

YGL1 8 AMAASD--TFRMPRFEVRDLESLEGKYDIVVCLDVLIHYPRFEAKQMIRHLASLAEKRVLISFAPRT 259 Yg 118 AMAASD--TFRMPREEVRDLESLEGKYDIVVCFDVLIHYPREEAKQMIRHLASLAEKRVLISFAPRT 259 SbChIM TAPGST--TFRMPRFEVRDLESLEGRYDVVVCLDVLIHYPREEAKAMIRHLASLADKRLLISFAPRT 263 ZMCh IM AQSSSS--SFRMPRFEVRDLESLEGRYDVVVCLDVLIHYPREEARAMIRHLASLADKRLLISFAPRT 258 NtCh $1 M$ KDKDDQSPAPVMPKEEVKDLESLDGKYDTVVCLDVMIHYPOSKADGMIAHLASLAENRLILSFAPKT 263 VVChIM SGRDDGLPAPVMPKEEVKDLESLDGKYHTVVCLDVLIHYPQSKADGMIAHLASLAEKRLILSFAPKT 266 PtChIM AGSEKE---LVMPKFEVKDLESLNGKYDTVVCLDVLIHYPQSKADGMIAHLASLAFNRLLLSFAPKT 255 GmChIM TSEDGS--GPVMPKFVVKDLESLDGKYDTVVCLDVLIHYPQSKADGMIAHLASLANKRLILSFAPKT 263 AtChIM SE--------NLPKFEVNDLESITGKYDTVVCLDVLIHYPQNKADGMIAHLASLAEKRVILSFAPKT 250 SYChIM YG--------NQPT FMTQDLAQLGGKYDTVICLDVLIHYPTEEASAMISHLASLADRRLILSFAPKT 168 CrCh IM AGQGKAP- -KVAPKFEALDLESVKGKYDTVTCLDVMIHYPQDKVDAMITHLAGLSDRRLIISFAPKT 264

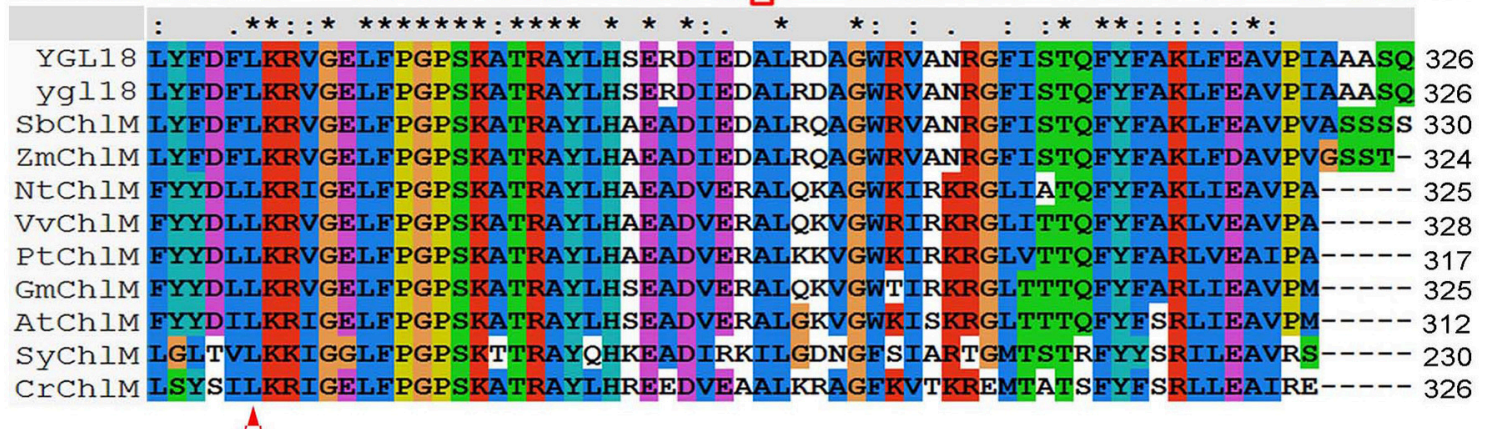
A

FIGURE 5 | Multiple sequences alignment of YGL18 and ygl18 with their homologs in different photosynthetic organisms. The amino acid substitution of Leu (L) to Phe (F) at position 225 in ygl18 protein sequences is labeled in a red rectangular box. The Leu (L) at position 270 in CrChIM, which is substituted in ch/M-1 in

(Continued) 
FIGURE 5 | Continued

Chlamydomonas reinhardtii (Meinecke et al., 2010), is indicated by the red triangle at the bottom. Three conserved cysteine residues for the redox regulation of ChIM activity (Richter et al., 2016), are indicated by the red number 1, 2, and 3, respectively, at the top. "*” fully conserved residue; ":" conservation between groups with strongly similar properties; "." conservation between groups with weakly similar properties; "-" gap. VvChIM, Vitis vinifera (XP_002280872.1); NtChIM, Nicotiana tabacum (AAF70243.1); PtChIM, Populus trichocarpa (XP_002318168.2); GmChIM, Glycine max (XP_003532350.3); AtChIM, Arabidopsis thaliana (NP_194238.1); OsChIM (YGL18), Oryza sativa (XP_015641356.1); SbChIM, Sorghum bicolor (XP_002436414.1); ZmChIM, Zea mays (AFW75394.1); CrChIM, Chlamydomonas reinhardtii (EDO97000.1); SyChIM, Synechocystis sp. PCC 6803 (BAA10812.1).

used to conduct the enzymatic reactions (Figure S5, lanes $5-7)$.

In the pathway of chlorophyll synthesis, ChlM catalyzes the formation of MgPME from MgP (Beale, 2005; Richter et al., 2013). In this study, contents of MgPME and MgP in the enzymatic assay were determined by HPLC. After the enzymatic progression, the synthesis of MgPME from $\mathrm{MgP}$ was observed with the GST-YGL18 protein (Figure 6B), but not with the GST control (Figure 6A). Interestingly, GST-ygl18 failed to catalyze the enzymatic reaction at first (Figure 6C). However, when a 4 fold GST-ygl18 amount was added into the reaction, a small quantity of MgPME was produced (Figure 6D). These results suggested that the YGL18 protein indeed performs ChlM enzymatic activity, but the mutation of YGL18 to ygl18 substantially weakens this enzymatic function.

To identify the attenuated ChlM function in the yglls mutant, the MgPME and MgP contents were further measured in 15-dayold wild-type and yglis plant leaves $\left(28^{\circ}, 8 \mathrm{~h}\right.$ light) by HPLC (Figures 7A,B). As expected, the MgP content of $1,524.03 \pm$ $81.28 \mathrm{pmol} \mathrm{g}^{-1} \mathrm{FW}$ in $y g l 18$ greatly exceeded that of $39.96 \pm$ $9.16 \mathrm{pmol} \mathrm{g}^{-1} \mathrm{FW}$ in the wild type; by contrast, the MgPME content of $44.65 \pm 6.13 \mathrm{pmol} \mathrm{g}^{-1} \mathrm{FW}$ in $y g l 18$ was almost 3 fold less than that of $119.33 \pm 6.44 \mathrm{pmol} \mathrm{g}^{-1} \mathrm{FW}$ in wild type (Figure 7C). These results supported the fact that the mutation of YGL18 to ygl18 makes the ChlM enzymatic function notably weakened, thus leading to the accumulation of the substrate MgP and the reduction of the product MgPME in the $y g l 18$ mutant leaves.

\section{YGL18 Is Required For Light-Dependent Chlorophyll Synthesis during the Greening of Etiolated Plants}

During the greening of etiolated plants, not every gene in the chlorophyll-synthesis pathway is required for light-dependent chlorophyll synthesis to occur. Previous studies have showed that PGL (OsCAO1) is required but FGL $(O s P O R B)$ is not essential for the process (Sakuraba et al., 2013; Yang et al., 2016). This differentiation prompted us to investigate the contribution of YGL18 to chlorophyll synthesis. When 6-dayold etiolated wild-type and ygl18 seedlings were exposed to light, the former quickly turned green, whereas the latter remained etiolated (Figure 8A). Correspondingly, chlorophyll contents increased rapidly in the etiolated wild-type seedlings, but this happened very slowly in the etiolated ygl18 seedlings (Figure 8B). Further examination of the YGL18 gene expression revealed it was rapidly upregulated in etiolated wild-type and ygl18 seedlings after illumination (Figure 8C). Together, these results suggested that YGL18 plays an essential role in the lightdependent chlorophyll synthesis during the greening of etiolated plants.

\section{YGL18 Is Required for Photoperiod-Regulated Chlorophyll Synthesis}

Surprisingly, the $y g l 18$ plants showed different leaf colors under varied photoperiod conditions. When the seedlings grew under the short-day $(8 \mathrm{~h})$, long-day $(14 \mathrm{~h})$ and continuous $(24 \mathrm{~h})$ light conditions, the $y$ gl18 leaf colors were yellow, yellow-green, and pale green, respectively (Figure 9A). The corresponding chlorophyll contents were measured subsequently. They were lowest for the short-day but increased gradually for the longday and continuous light conditions in ygl18 leaves (Figure 9B). Meanwhile, the chlorophyll $a / b$ ratio was highest for the shortday but decreased gradually for the long-day and continuous light conditions in $y g l 18$ leaves (Figure 9C). In addition, we tested the daily expression patterns of the YGL18 gene in wildtype and $y g l 18$ seedling leaves under the short-day and longday conditions. Under both conditions, the YGL18 expression levels in the wild-type and ygl18 seedling leaves were upregulated by light yet downregulated by dark (ons, the ygl18 leaf colors were yellow, yellow-green, and pale green, respectively (Figures 9D-G). Taken together, these results suggested that the YGL18 gene is required for photoperiod-regulated chlorophyll synthesis.

\section{YGL18 Is Required for Plant Growth and Survival under High Light Conditions}

The ygl18 mutants showed severely stunted growth, and even death, when planted in Wuhan of Hubei province, but they grew similarly to the wild type when planted in Lingshui of Hainan province (Figures 1D,E). It would be interesting to know the reason for this growth difference. Under natural field conditions, a longer day length, higher temperature and higher solar radiation occurred at the planting time in Wuhan than in Lingshui (Figure S6). Accordingly, we explored the growth status of the wild-type and $y g l 18$ plants under different experimental conditions in the plant growth chamber. When the wild-type and $y g l 18$ plants were cultivated under a 14 -h light condition $\left(190 \mu \mathrm{mol} \mathrm{m} \mathrm{m}^{-2} \mathrm{~s}^{-1}, 26 / 19^{\circ} \mathrm{C}\right.$ for light/dark), mimicking the day length in Wuhan, the $y g l 18$ mutant grew to a similar height as the wild type (Figures 10A,B). Based on this growth condition, the growth temperature was further raised to $31 / 22^{\circ} \mathrm{C}$ and $35 / 22^{\circ} \mathrm{C}$ (14-h light, $190 \mu \mathrm{mol} \mathrm{m} \mathrm{m}^{-2}$ $\left.\mathrm{s}^{-1}\right)$, but the $y g l 18$ plants maintained equivalent growth with 

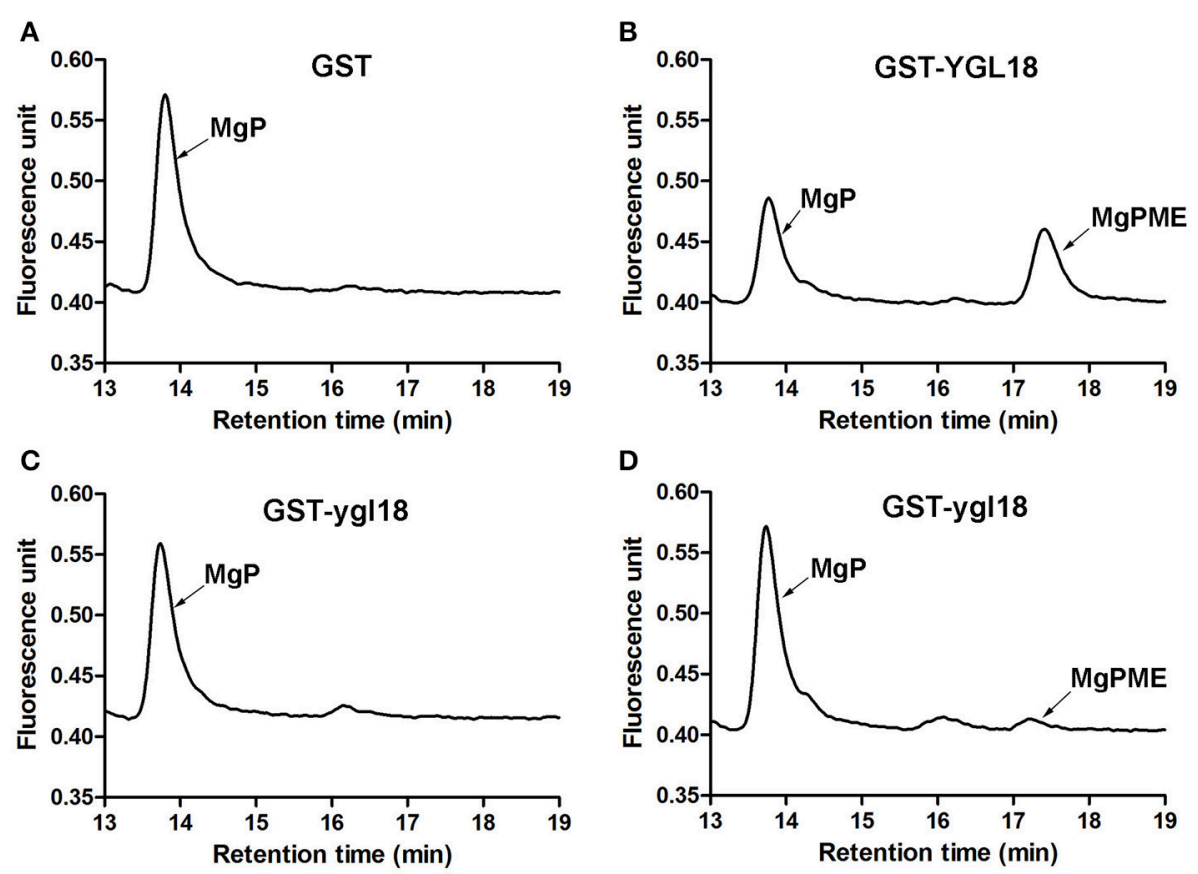

FIGURE 6 | ChIM enzymatic activities of YGL18 and ygl18 based on HPLC. The enzymatic assays were conducted by adding different purified proteins: (A) GST (0.6 $\mu \mathrm{M})$; (B) GST-YGL18 (0.6 $\mu \mathrm{M})$; (C) GST-ygl18 (0.6 $\mu \mathrm{M})$; (D) GST-ygl18 $(2.4 \mu \mathrm{M})$. The arrows indicate the peak positions of the substrate MgP (about $13.7 \mathrm{~min})$ and the product MgPME (about $17.3 \mathrm{~min}$ ), which are distinguished by comparing with the standard curve (Figure S2). Different letters indicate values are statistically different based on a one-way ANOVA analysis.

the wild type (Figures 10C-F). Next, we tried to enhance the light intensity. The ygl18 plants still grew to a similar height as the wild type when the light intensity was set to $500 \mu \mathrm{mol}$ $\mathrm{m}^{-2} \mathrm{~s}^{-1}$ (14-h light, $31 / 22^{\circ} \mathrm{C}$ ) (Figures 10G,H). However, when the light intensity was enhanced to $900 \mu \mathrm{mol} \mathrm{m}{ }^{-2} \mathrm{~s}^{-1}$ (14-h light, $31 / 22^{\circ} \mathrm{C}$ ), the $y g l 18$ plants did not grow as well as the wild type (Figures 10I,J). Therefore, it is inferred that it was the higher light intensity that caused the retarded growth of ygl18 in Wuhan, but neither its longer day length nor higher temperature.

We also explored the growth status of wild-type and ygl18 plants under three different natural field conditions. These three conditions in the planting field were emerged by the solar motion combining with tree shadow, resulting in the sunlight time and light intensity lowest for condition 1, moderate for condition 2, and highest for condition 3 (Figure 11A). Eight-day-old seedlings were simultaneously planted in the fields under these three light conditions (Figure 11B). Then, they encountered one cloudy day and subsequent 18 sunny days. After 9 days in sunlight (DIS), the ygl18 mutant showed yellow-green leaves under condition 1, yellow leaves under condition 2, and yellow-tending-towhite leaves under condition 3 (Figure 11B). Correspondingly, under conditions 1,2, and 3, the chlorophyll contents were gradually decreased in ygl18 but remained steady and stable in wild-type seedlings after nine DIS (Figure 11C). Surprisingly, after 18 DIS, the $y g l 18$ mutants showed yellow leaves under condition 1 , but yellow-tending-to-white and partially withered leaves under condition 2, and totally withered and dead leaves under condition 3 (Figure 11B). In addition, because of the high light intensity, the growth of all the $y g l 18$ plants was markedly reduced under these three conditions, as we expected (Figure 11D).

Taken together, ygl18 showed impeded growth under high light intensity. When the light intensity was sufficiently high, the ygl18 seedlings were more susceptible to die at this light intensity or after longer exposure to it. It is suggested that YGL18 plays an essential role in plant growth and survival under high light conditions.

\section{DISCUSSION}

\section{The YGL18 Gene Is Cloned as the ChIM Gene in the Monocotyledonous Plant-Rice}

The ChlM gene is one of the key genes in the chlorophyll synthesis pathway. Its mutants have ever only been found in Arabidopsis (Pontier et al., 2007) and Chlamydomonas reinhardtii (Meinecke et al., 2010). And its enzymatic activities have been well studied in Synechocystis sp. PCC6803 (Shepherd et al., 2003; Shepherd and Hunter, 2004), tobacco (Alawady et al., 2005), and Arabidopsis (Block et al., 2002; Richter et al., 2013, 2016). To date, however, no ChlM gene has yet been identified and studied in monocotyledonous plants. In the present study, a spontaneous yellow-green-leaf rice 


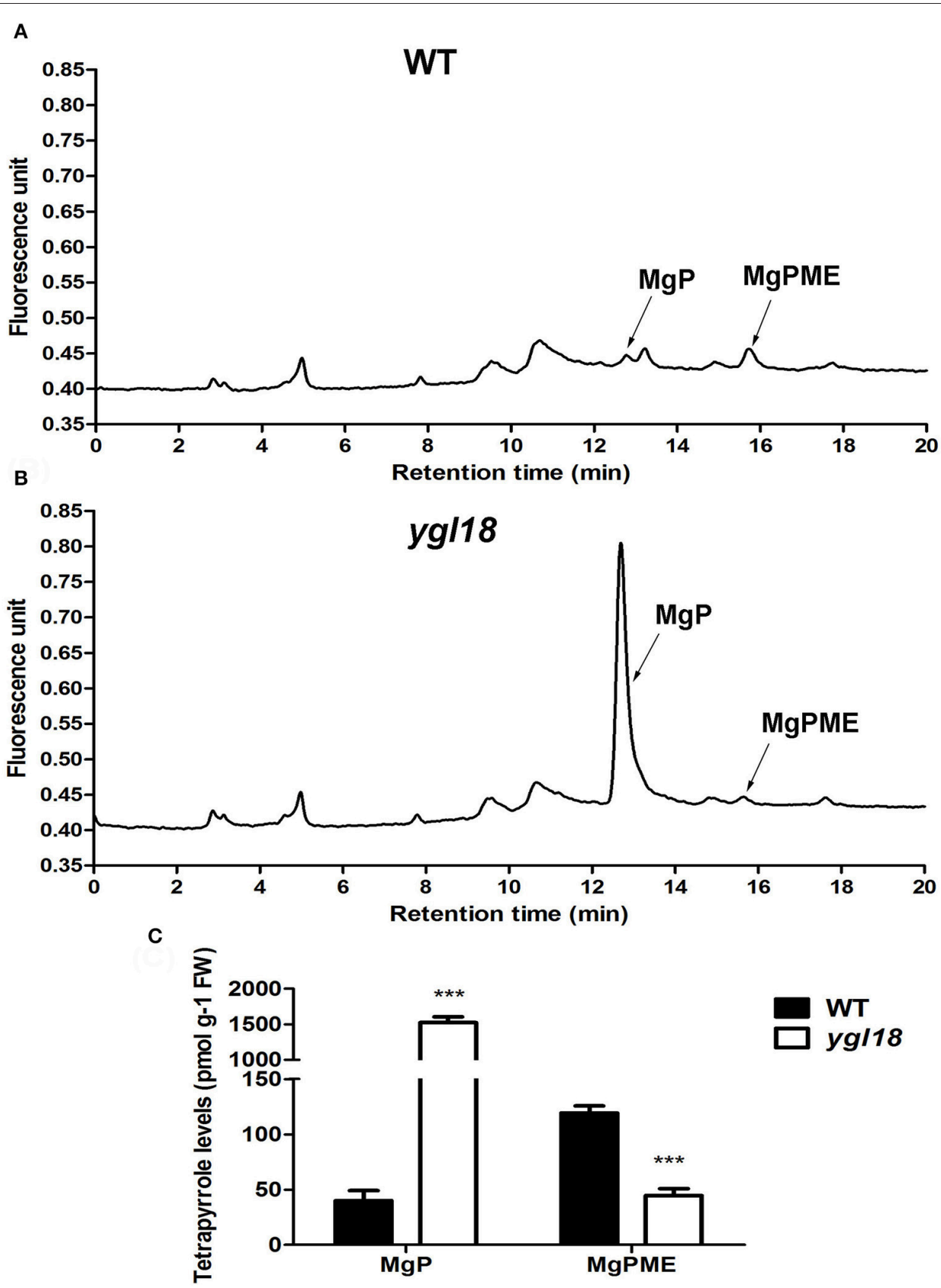

FIGURE 7 | HPLC analysis of MgP and MgPME contents from the seedling leaves of wild-type and yg/18 plants. (A,B) Representative chromatograms of the extracts from wild-type (A) and yg/18 (B) plants $\left(28^{\circ} \mathrm{C}\right.$, $8 \mathrm{~h}$ light condition, $\left.150 \mu \mathrm{mol} \mathrm{m}{ }^{-2} \mathrm{~s}^{-1}\right)$. The arrows indicate the peak positions of MgP (about $\left.12.7 \mathrm{~min}\right)$ and $\mathrm{MgPME}$ (about $15.8 \mathrm{~min}$ ), which are distinguished by comparing with the standard curve (Figure S3). (C) Quantification of MgP and MgPME contents. FW, fresh weight. Data represent the mean \pm SD of three biological replicates (Student's $t$-test: ${ }^{\star \star \star} P<0.0005$ ). Different letters indicate values are statistically different based on a one-way ANOVA analysis.

mutant, $y g l 18$, was successfully isolated (Figure 1), and the YGL18 gene was cloned (Figure 2), that encodes a protein homologous with the AtChlM (Figure 5 and Figure S4). The recombinant YGL18 protein expressed in E. coli was shown to perform ChlM enzymatic activity (Figure 6). Therefore, we succeeded in identifying a ChlM gene in a monocotyledonous plant.
The Rice YGL18 Protein Is a Functionally Conserved ChIM Protein, and the Substitution of a Conserved Leu to Phe in ygl18 Starkly Weakens Its Function

The amino acid residues of YGL18 and other ChlM orthologues from different photosynthetic organisms are highly conserved 

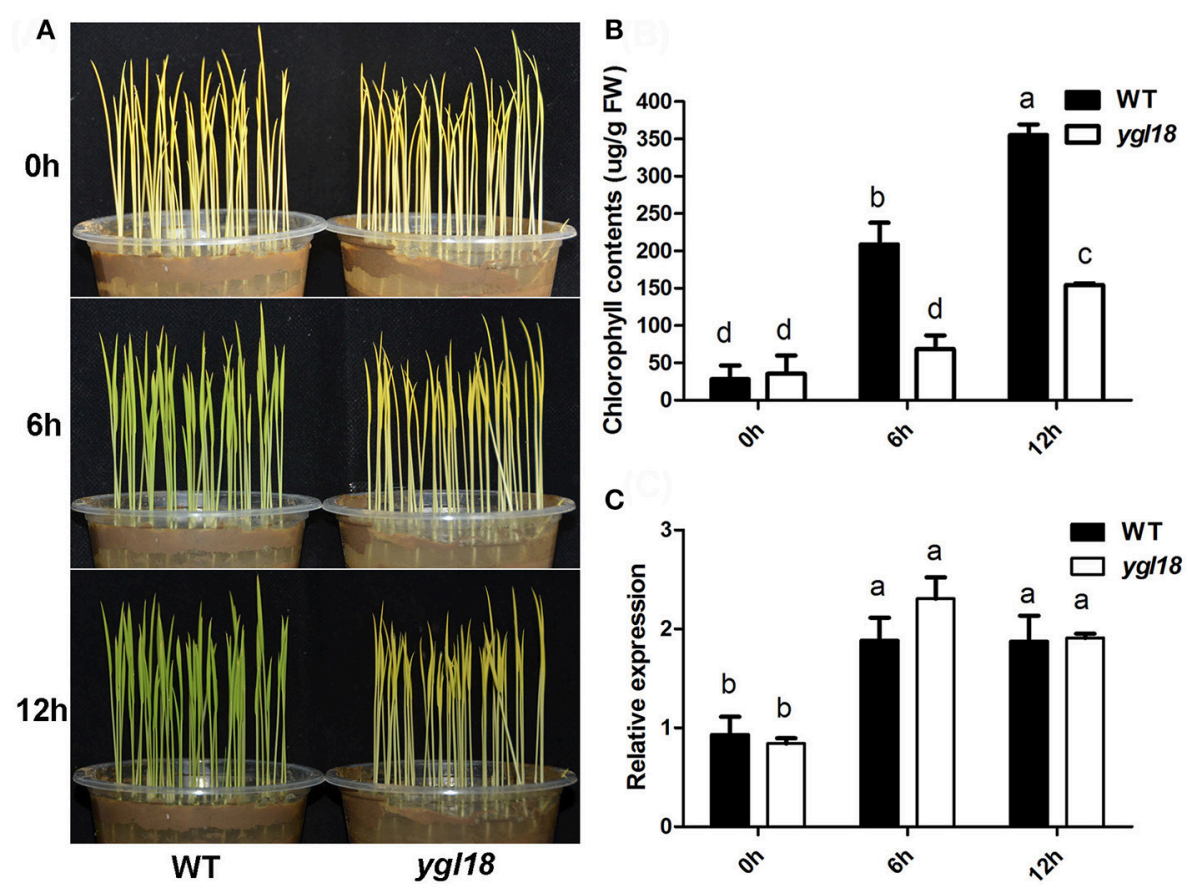

FIGURE 8 | Expression levels of YGL18 during the greening of etiolated seedlings. The 6-day-old etiolated seedlings of the wild type and ygl18 were exposed to light (150 $\mu \mathrm{mol} \mathrm{m} \mathrm{m}^{-2} \mathrm{~s}^{-1}$ ) for 0-12 h. (A) Comparison of the greening speed between the wild-type and yg/18 seedlings. (B) Chlorophyll contents in the wild-type and yg/18 seedlings during greening. (C) Changes in transcript levels of YGL18 in the wild-type and yg/18 seedlings during greening. In (B,C), data represent the mean \pm SD of three biological replicates (one-way ANOVA analysis); FW, fresh weight. Different letters indicate values are statistically different based on a one-way ANOVA analysis.

(Figure 5). These conserved sequences belong to the conserved protein domain "BchM-ChlM" (TIGR02021), which represents the $S$-adenosylmethionine-dependent $\mathrm{O}$-methyltransferase responsible for the methylation of $\mathrm{MgP}$ (Marchler-Bauer et al., 2015). These conserved amino acid residues may be essential for the ChlM function. A missense mutation of chlM-1 in Chlamydomonas reinhardtii made these mutants incapable of accumulating chlorophyll (Meinecke et al., 2010), where the mutation resulted in a replacement of a leucine (Leu) by a proline (Pro) residue at position 270 in CrChlM, meanwhile, Leu at this particular position remained completely conserved in our analysis (Figure 5). In this study, the Leu at position 225 in the YGL18 protein is also originally a fully conserved amino acid residue among the analyzed organisms, but it is substituted by Phe in the ygl18 protein (Figure 5). It is speculated that this Leu may play an important role for the OsChlM protein function. Previous study has indicated that the amino acid of ChlM proteins corresponding to the Leu at position 225 in the YGL18 protein is involved in binding to the cofactor SAM for ChlM proteins in different organisms (Chen et al., 2014). In addition, it was recently proposed that the NADPH-dependent thioredoxin reductase $\mathrm{C}$ has a regulatory impact on the redox status of conserved cysteine residues of ChlM, thus altering the ChlM activity (Richter et al., 2013). Further research has identified three conserved cysteine residues essential for the catalytic function and the redox-dependent activation of the Arabidopsis ChlM (Richter et al., 2016). These three cysteine residues are also conserved in the YGL18 protein (Figure 5), implying their possible functions on the redox regulation of ChlM enzymatic activity in rice. Interestingly, the third cysteine is just located at the left neighboring site of Leu 225 of YGL18 (Figure 5), meaning that the Leu at position 225 is also probably involved in redox regulation of ChlM enzymatic activity. Therefore, it is plausible that the amino-acid substitution of Leu to Phe in the ygl18 protein may impact the ChlM activity by changing its binding or catalyzing ability to SAM.

ChlM catalyzes the formation of MgPME from MgP in the chlorophyll synthesis pathway (Richter et al., 2013). In this study, the YGL18 protein was also verified to perform the ChlM activity (Figure 6B), just as the ChlM proteins do in Synechocystis sp. PCC6803 (Shepherd et al., 2003; Shepherd and Hunter, 2004), Arabidopsis (Block et al., 2002), and tobacco (Alawady et al., 2005). This clearly confirms that the YGL18 gene is the corresponding ChlM gene in rice. The in vitro assay revealed that the ChlM enzymatic activity of the ygl18 protein was greatly reduced, with the slight synthesis of $\mathrm{MgPME}$ from $\mathrm{MgP}$ requiring a large amount of the ygl18 protein (Figures 6C,D). Further, given that the MgPME content could be measured in the $y g l 18$ leaves (Figures $7 \mathrm{~B}, \mathbf{C}$ ), this supports the conclusion that the single amino acid exchange from Leu to Phe in ygl18 did not completely negate the protein's biological function. Most possibly, it is just the remained slight activity of the ygl18 protein that results in the characteristics of $y g l 18$ mutant on chlorophyll synthesis and plant growth, as discussed later. 

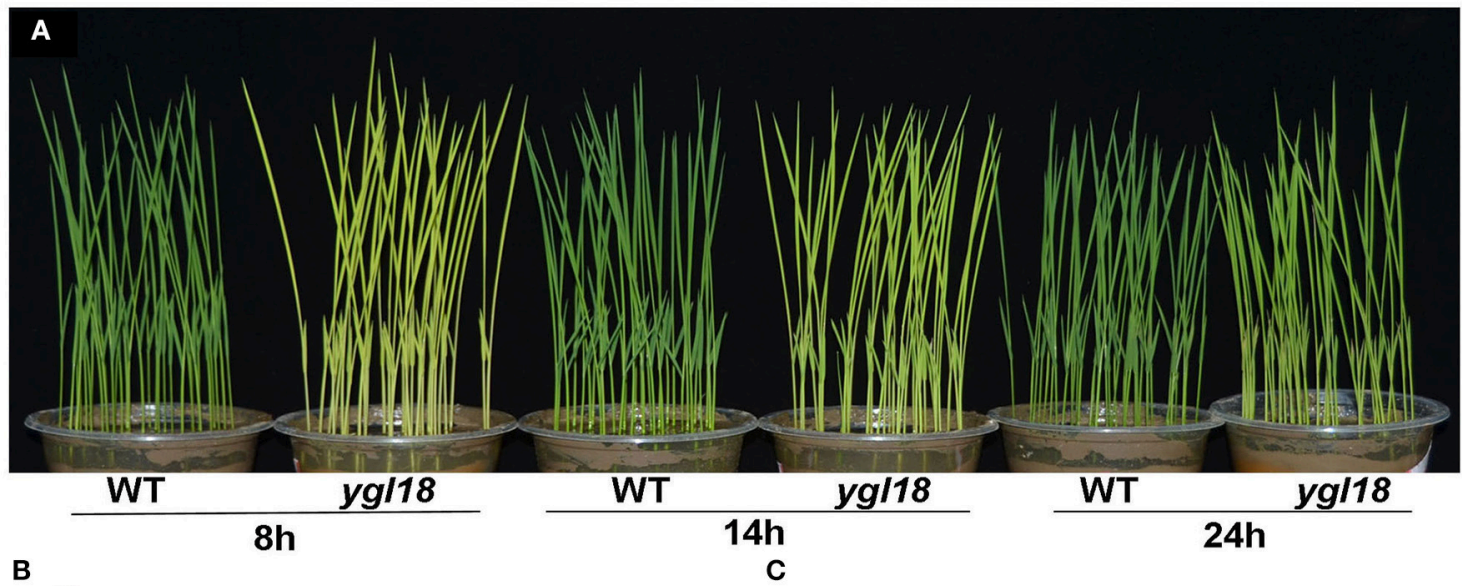

B
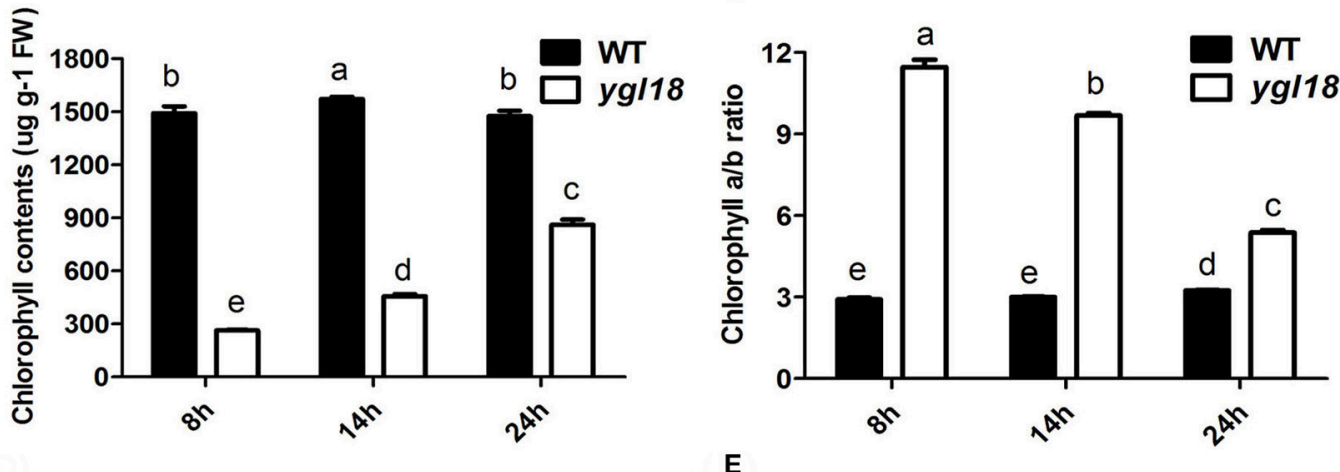

D
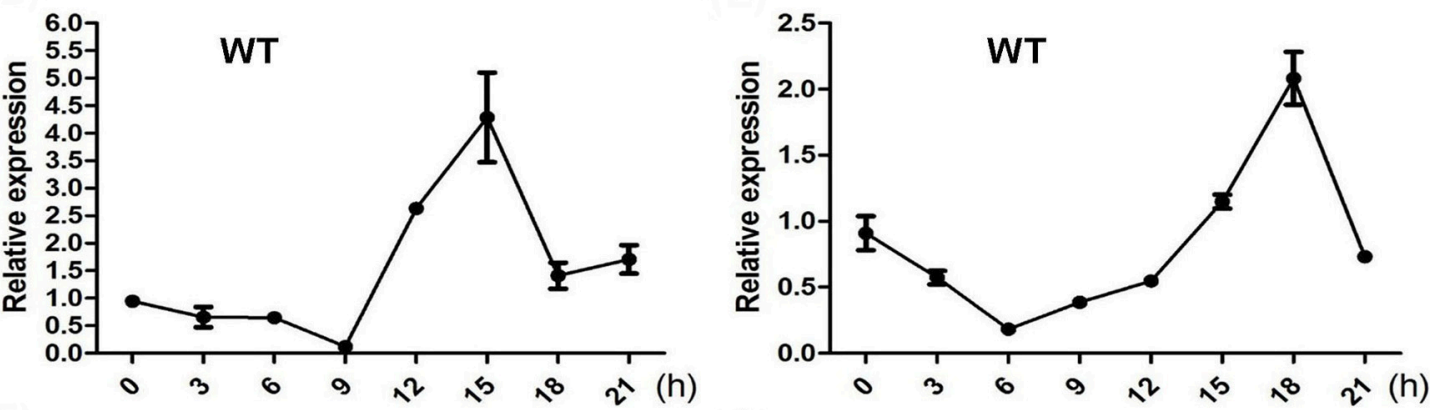

$\mathbf{F}$
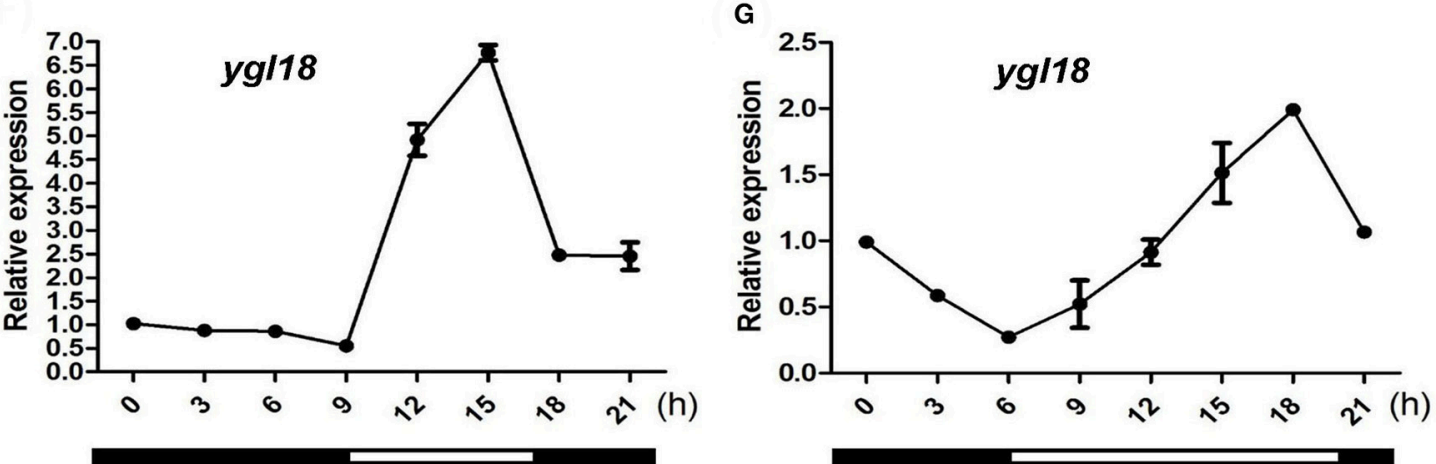

FIGURE 9 | Photoperiod-dependent chlorophyll synthesis in yg/18 leaves. (A) Leaf phenotypes of the 12-day-old wild-type and yg/18 seedlings under short-day (8 $\mathrm{h}$ ), long-day (14 h) and continuous (24 h) light conditions. (B) Chlorophyll contents of plant leaves in (A). FW, fresh weight. (C) Chlorophyll a/b ratio of the plant leaves in (A). In (B,C), data represent the mean \pm SD of four biological replicates (one-way ANOVA analysis). (D-G) Daily expression patterns of YGL18 in the wild-type and ygl18 seedling leaves under the short-day (D,F, $8 \mathrm{~h}$ light: 9:00 to 17:00.) and the long-day conditions (E,G, 14 h light: 6:00 to 20:00.). Leaf samples were harvested at $3 \mathrm{~h}$ intervals. Data represent the mean $\pm \mathrm{SD}$ of two biological replicates. Different letters indicate values are statistically different based on a one-way ANOVA analysis. 

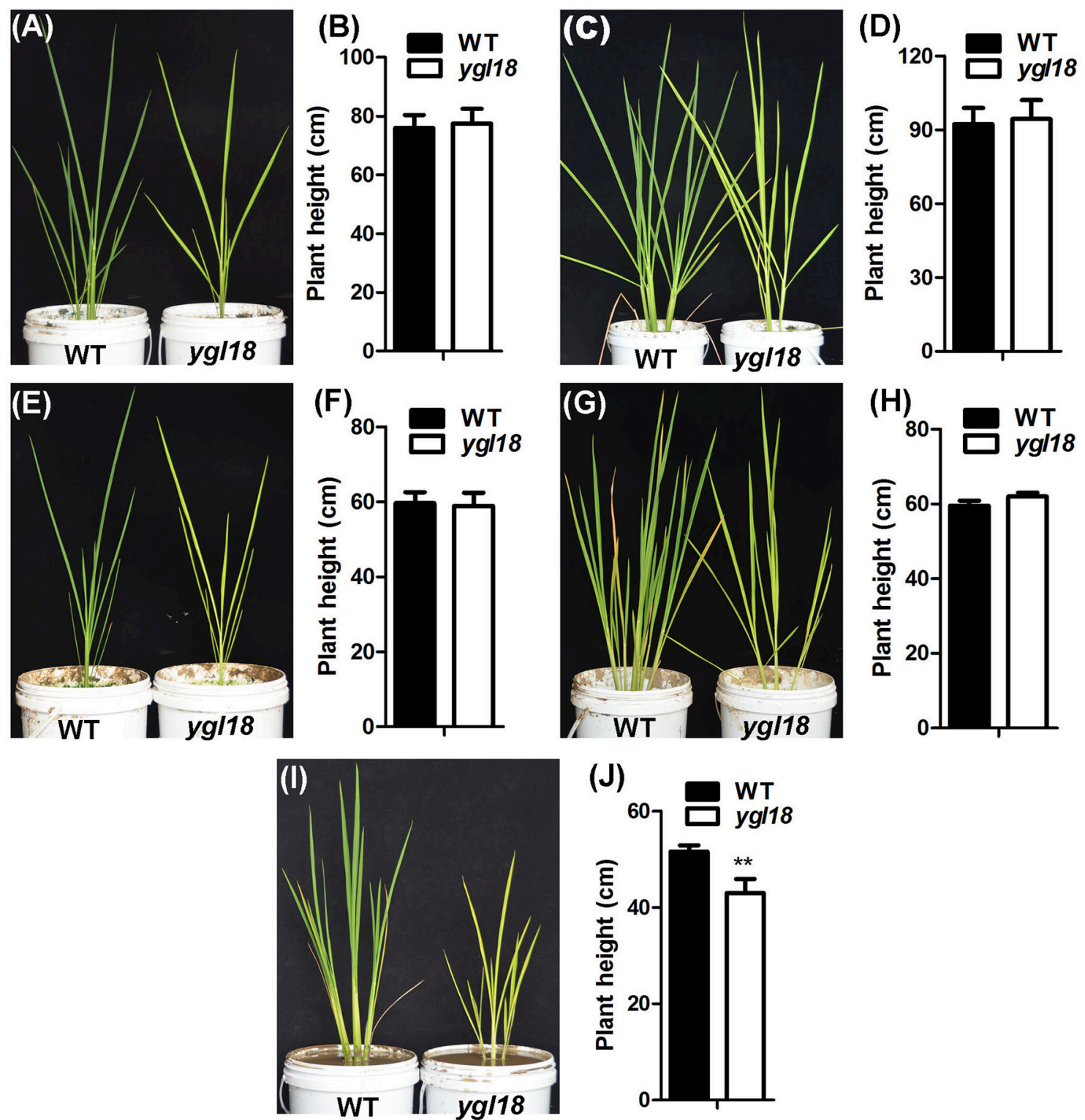

FIGURE 10 | Growth of the wild-type and $y g / 18$ plants under different experimental conditions in the plant growth chamber. Fifteen-day-old seedlings ( $28 / 22^{\circ} \mathrm{C}$, $14 / 10 \mathrm{~h}$, light/dark, $190 \mu \mathrm{mol} \mathrm{m}{ }^{-2} \mathrm{~s}^{-1}$ ) were used for the following experimental conditions. (A) Plants grown for about 60 days under the condition of $26 / 19^{\circ} \mathrm{C}$ 14/10 h, $190 \mu \mathrm{mol} \mathrm{m}^{-2} \mathrm{~s}^{-1}$. (B) Plant height under the condition in (A). (C) Plants grown for about 60 days under the condition of $31 / 22^{\circ} \mathrm{C}, 14 / 10 \mathrm{~h}, 190 \mu \mathrm{mol} \mathrm{m}^{-2}$ $\mathrm{s}^{-1}$. (D) Plant height under the condition in (C). (E) Plants grown for about 30 days under the condition of $35 / 22^{\circ} \mathrm{C}, 14 / 10 \mathrm{~h}, 190 \mu \mathrm{mol} \mathrm{m}^{-2} \mathrm{~s}^{-1}$. (F) Plant height under the condition in (E). (G) Plants grown for about 30 days under the condition of $31 / 22^{\circ} \mathrm{C}, 14 / 10 \mathrm{~h}, 500 \mu \mathrm{mol} \mathrm{m}^{-2} \mathrm{~s}^{-1}$. (H) Plant height under the condition in (G). (I) Plants grown for about 23 days under the condition of $31 / 22^{\circ} \mathrm{C}, 14 / 10 \mathrm{~h}, 900 \mu \mathrm{mol} \mathrm{m}^{-2} \mathrm{~s}^{-1}$. (J) Plant height under the condition in (I). Data represent the mean \pm SD of three independent plants (Student's $t$-test: ${ }^{\star \star} P<0.005$ ).

\section{The Rice YGL18 (ChIM) Gene Is Required for Light-Dependent and Photoperiod-Regulated Chlorophyll Synthesis}

The YGL18 gene is mainly expressed in green tissues, especially in leaves (Figure 3), where the YGL18 protein is localized in the chloroplasts (Figure 4). These results are all consistent with the fact that the ChlM gene encodes one of the key enzymes in the chlorophyll synthesis pathway. Among the cloned genes in the chlorophyll synthesis pathway, the PGL (OsCAO1) gene was identified as required for light-dependent chlorophyll synthesis during the greening of the etiolated plants (Yang et al., 2016), but the FGL (OsPORB) gene was verified as not required for this biological process (Sakuraba et al., 2013). In this study, the YGL18 (ChlM) gene was clearly essential for 


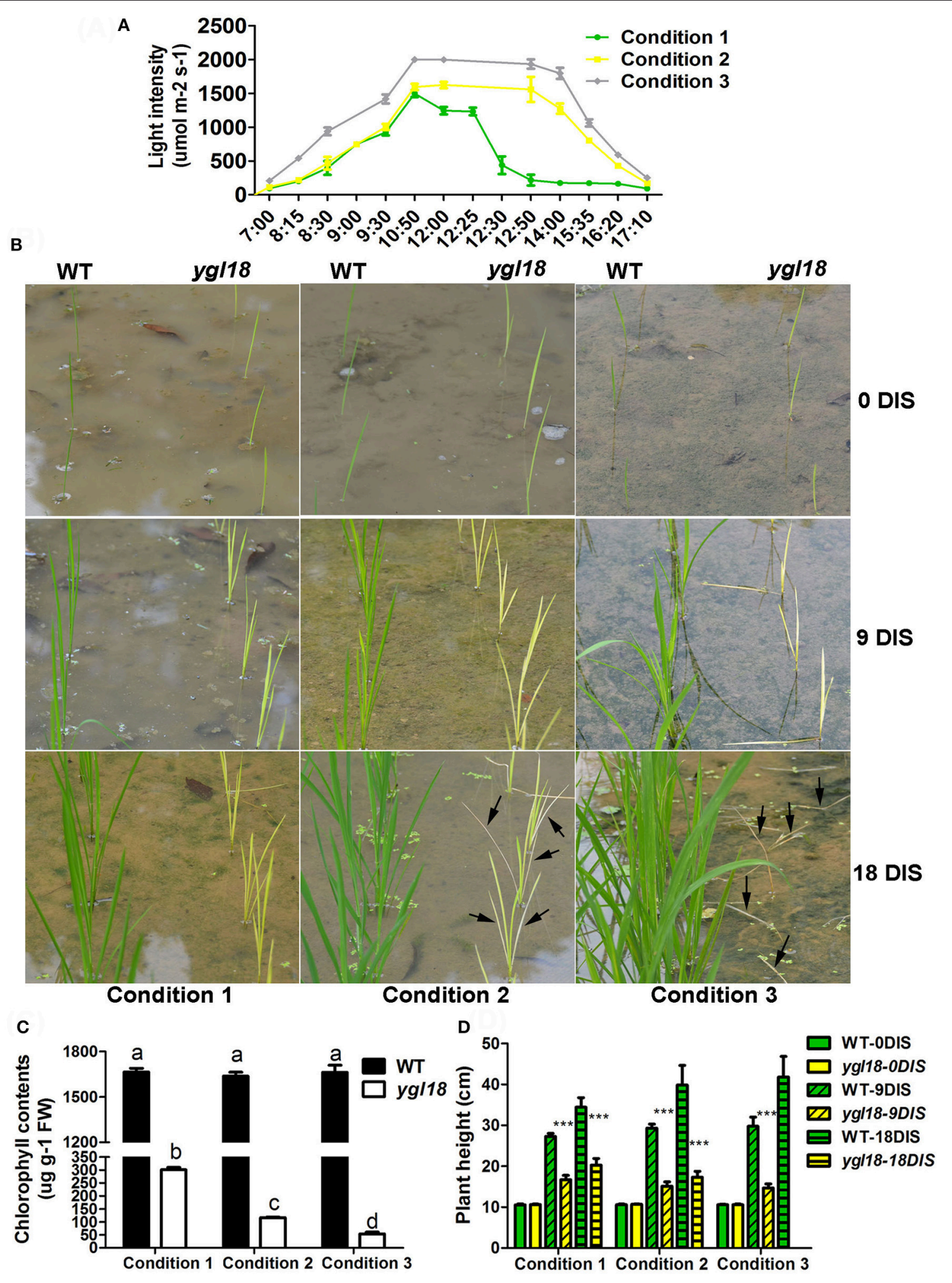

FIGURE 11 | Growth of the wild-type and yg/18 plants under different light conditions in the natural field. (A) Three different light conditions were generated by the solar motion and the tree shadow. Condition 1 had about 4 h' time in sunlight (8:30-12:30), about $3 h^{\prime}$ light intensity over 1,000 $\mu$ mol $\mathrm{m}^{-2} \mathrm{~s}^{-1}$, and a peak light intensity of about $1,500 \mu \mathrm{mol} \mathrm{m} \mathrm{m}^{-2} \mathrm{~s}^{-1}$. Condition 2 had about $8 \mathrm{~h}$ ' time in sunlight (8:30-16:20), about $5 \mathrm{~h}^{\prime}$ light intensity over $1,000 \mu \mathrm{mol} \mathrm{m}^{-2} \mathrm{~s}^{-1}$, and a peak light intensity of about $1,600 \mu \mathrm{mol} \mathrm{m} \mathrm{m}^{-2} \mathrm{~s}^{-1}$. Condition 3 had about $8 \mathrm{~h}$ ' time in sunlight (8:15-16:20), about $7 \mathrm{~h}$ ' light intensity over $1,000 \mu \mathrm{mol} \mathrm{m}^{-2} \mathrm{~s}^{-1}$, and a 
FIGURE 11 | Continued

peak light intensity of over 2,000 $\mu \mathrm{mol} \mathrm{m} \mathrm{m}^{-2} \mathrm{~s}^{-1}$. (B) Growth status of the wild-type and $y g / 18$ plants under above three light conditions. Eight-day-old seedlings were planted in the fields and encountered one cloudy day and subsequent 18 sunny days (condition 1,2, and 3). Plants were observed after 0,9 , and 18 days in sunlight (DIS). The black arrows indicate the withered leaves. (C) Chlorophyll contents of the wild-type and yg/18 plant leaves at 9 DIS. Data represent the mean \pm SD of three biological replicates (one-way ANOVA analysis); FW, fresh weight. (D) Plant height of the wild-type and yg/18 plants at 0, 9, and 18 DIS under above three conditions. Data represent the mean \pm SD of over eight independent plants (Student's $t$-test: ${ }^{* \star *} P<0.0005$ ). ND, not detected for dead plants. Different letters indicate values are statistically different based on a one-way ANOVA analysis.

light-dependent chlorophyll synthesis during the greening of the etiolated WT and $y g l 18$ plants (Figure 8). Impact of photoperiod on chlorophyll synthesis is rarely reported. More chlorophyll contents were accumulated in the $y g l 18$ leaves when grown under a longer light application time (Figures 9A,B). Similar phenomena were found from the chlB knockout mutant in liverwort (Marchantia polymorpha L.), where it was suggested that $c h l B$ was required for chlorophyll biosynthesis under short photoperiod (Ueda et al., 2014). Nevertheless, there may be a different explanation for this phenomenon in ygl18. The ygl18 protein did not completely cancel the protein function, and a large amount of the ygl18 protein could catalyze the formation of a small quantity of MgPME (Figure 6D). In addition, the circadian expression patterns of the YGL18 gene revealed that it was up-regulated by light yet down-regulated by dark conditions (Figures 9D-G). As a result, under longer light conditions, the yglis mutant possibly produced more ygl18 proteins for the enzymatic catalyzation, and thus eventually accumulated more chlorophyll in its leaves. Taken together, the results strongly suggested that the YGL18 (ChlM) gene plays an essential role in light-dependent and photoperiod-regulated chlorophyll synthesis.

\section{The Rice YGL18 (ChIM) Gene Is Essential for Plant Growth and Survival under High Light Conditions}

Scarce are studies that pay in-depth attention to the effect on plant growth from functional genes in the chlorophyll synthesis pathway. It was surprising that $y g l 18$ showed severely retarded growth, and even death, in the paddy field in Wuhan, but nearnormal growth in Lingshui (Figure 1). When the wild-type and ygl18 plants were cultivated in the plant growth chamber under different temperature and light conditions, it was the high light intensity, but not the long day length or the high temperature, which impaired the $y g l 18$ growth (Figure 10). Field experiments with different sunlight time and light intensity further showed that the $y g l 18$ plants were more prone to death under a higher light intensity and a longer exposure time to it (Figure 11). Effects of light intensity upon chlM mutants have also been reported for other species. In Chlamydomonas reinhardtii, chlM1 has an amino-acid substitution, while chlM-2 do not produce the ChlM protein; consequently, chlM-1 can survive under the light intensity of $45 \mu \mathrm{mol} \mathrm{m} \mathrm{m}^{-2} \mathrm{~s}^{-1}$, but not under a higher light intensity of $500 \mu \mathrm{mol} \mathrm{m} \mathrm{m}^{-2} \mathrm{~s}^{-1}$, while chlM-2 can only weakly survive under $1.6 \mu \mathrm{mol} \mathrm{m}^{-2} \mathrm{~s}^{-1}$ of light (Meinecke et al., 2010). The Arabidopsis chlM mutant lacks the ChlM mRNA and ChlM protein because of the T-DNA insertion; hence, its mutant seedlings turned white and stopped growing after cotyledon development when germinated under the light intensity of 70 $\mu \mathrm{mol} \mathrm{m}{ }^{-2} \mathrm{~s}^{-1}$ (Pontier et al., 2007). Apparently, the complete loss of ChlM function is lethal for plants to grow under well-lit conditions, while an impaired ChlM function makes the plants survival only under low light intensity conditions. Meanwhile, MgP, the substrate of ChlM, notably accumulated in Chlamydomonas reinhardtii and Arabidopsis chlM mutants, as described. Similarly, we found that $\mathrm{MgP}$ also remarkably accumulated in the rice $y g l 18$ mutants (Figures 7B-C). However, rice mutants, which were related to genes in the chlorophyll synthesis pathway, such as $y g l 7$ (Deng et al., 2014), ygll (Wu et al., 2007), and pgl (Yang et al., 2016), grew only slightly lower than their wild type, and these mutants still contained plenty of chlorophyll thus showed near-green leaves. It was also the fact that the intermediate metabolites of chlorophyll synthesis reportedly did not substantially accumulate (about 2 -fold for $\mathrm{MgP}$, and $<2$-fold for chlide, the substrate of YGL1) in $y g l 1$ mutants. Considering these studies, it would appear that fluent synthesis of chlorophyll is essential for functional plant growth. The accumulation of the intermediate metabolites, like MgP, is probably the key factor to influence plant growth under high light conditions. When accumulated, such intermediate metabolites may induce oxidative damages under light irradiation, while photoautotrophic growth relying on chlorophyll synthesis may support plant resistance to this damages. It is speculated that a dynamic equilibrium between the damages induced by accumulated $\mathrm{MgP}$ and resistance originating from the leaky synthesis of chloroph1yll is critical for the growth of $y g l 18$ plants. To completely elucidate this scientific issue, a further detailed study will be needed.

\section{CONCLUSION}

In conclusion, this study identified the ChlM gene in rice plants. It is expressed mainly in the leaf tissue and its protein product, is localized in the chloroplasts to perform the biological roles. The enzymatic activity of ChlM was verified. ChlM is required for light-dependent and photoperiod-regulated chlorophyll synthesis. And also, ChlM plays an essential role in light intensity-related plant growth and survival dynamics.

\section{AUTHOR CONTRIBUTIONS}

ZW designed and participated in all the experimental procedures, performed the data analysis and also drafted the manuscript; $\mathrm{XH}$ participated in the gene cloning and complementary experiments; $\mathrm{KH}$ performed the gene expression experiments; YW helped to prepare the figures and did partial writing; XW and 
SD participated in planting the rice materials; YL participated in the vector construction; $\mathrm{DH}$ and $\mathrm{KC}$ participated in the sample preparation; BA participated in the figure preparation; YsL supervised the study and critically revised the manuscript. All authors read and approved the final manuscript.

\section{FUNDING}

This work was supported by the National Key Research and Development Program of China (2016YFD0100400), China Postdoctoral Science Foundation (2016M592379), the National Special Key Project for Transgenic Breeding (Grant No. 2016ZX08001001), the Doctoral Starting up Foundation (Jiangxi Agricultural University, 9232306135), and the Open Research

\section{REFERENCES}

Alawady, A. E., and Grimm, B. (2005). Tobacco Mg protoporphyrin IX methyltransferase is involved in inverse activation of $\mathrm{Mg}$ porphyrin and protoheme synthesis. Plant J. 41, 282-290. doi: 10.1111/j.1365-313X.2004.02291.x

Alawady, A., Reski, R., Yaronskaya, E., and Grimm, B. (2005). Cloning and expression of the tobacco CHLM sequence encoding $\mathrm{Mg}$ protoporphyrin IX methyltransferase and its interaction with Mg chelatase. Plant Mol. Biol. 57, 679-691. doi: 10.1007/s11103-005-1427-8

Beale, S. I. (2005). Green genes gleaned. Trends Plant Sci. 10, 309-312. doi: 10.1016/j.tplants.2005.05.005

Block, M. A., Tewari, A. K., Albrieux, C., Marechal, E., and Joyard, J. (2002). The plant S-adenosyl-L-methionine:Mg-protoporphyrin IX methyltransferase is located in both envelope and thylakoid chloroplast membranes. Eur. J. Biochem. 269, 240-248. doi: 10.1046/j.0014-2956.2001.02643.X

Chen, X., Wang, X., Feng, J., Chen, Y., Fang, Y., Zhao, S., et al. (2014). Structural insights into the catalytic mechanism of Synechocystis magnesium protoporphyrin IX O-methyltransferase (ChlM). J. Biol. Chem. 289, 25690-25698. doi: 10.1074/jbc.M114.584920

Czarnecki, O., and Grimm, B. (2012). Post-translational control of tetrapyrrole biosynthesis in plants, algae, and cyanobacteria. J. Exp. Bot. 63, 1675-1687. doi: $10.1093 / \mathrm{jxb} / \mathrm{err} 437$

Deng, X. J., Zhang, H. Q., Wang, Y., He, F., Liu, J. L., Xiao, X., et al. (2014). Mapped clone and functional analysis of leaf-color gene Ygl7 in a rice hybrid (Oryza sativa L. ssp. indica). PLoS ONE 9:e99564. doi: 10.1371/journal.pone.00 99564

Fromme, P., Melkozernov, A., Jordan, P., and Krauss, N. (2003). Structure and function of photosystem I: interaction with its soluble electron carriers and external antenna systems. FEBS Lett. 555, 40-44. doi: 10.1016/S0014-5793(03)01124-4

Grossman, A. R., Bhaya, D., Apt, K. E., and Kehoe, D. M. (1995). Lightharvesting complexes in oxygenic photosynthesis: diversity, control, and evolution. Annu. Rev. Genet. 29, 231-288. doi: 10.1146/annurev.ge.29.120195.0 01311

Jung, K. H., Hur, J., Ryu, C. H., Choi, Y., Chung, Y. Y., Miyao, A., et al. (2003). Characterization of a rice chlorophyll-deficient mutant using the T-DNA genetrap system. Plant Cell Physiol. 44, 463-472. doi: 10.1093/pcp/pcg064

Lee, S., Kim, J. H., Yoo, E. S., Lee, C. H., Hirochika, H., and An, G. (2005). Differential regulation of chlorophyll a oxygenase genes in rice. Plant Mol. Biol. 57, 805-818. doi: 10.1007/s11103-005-2066-9

Lichtenthaler, H. K. (1987). Chlorophylls and carotenoids: pigments of photosynthetic biomembranes. Meth. Enzymol. 148, 350-382. doi: 10.1016/0076-6879(87)48036-1

Marchler-Bauer, A., Derbyshire, M. K., Gonzales, N. R., Lu, S., Chitsaz, F., Geer, L. Y., et al. (2015). CDD: NCBI's conserved domain database. Nucleic Acids Res. 43, D222-D226. doi: 10.1093/nar/gku1221
Fund of State Key Laboratory of Hybrid Rice (Wuhan University, KF201402).

\section{ACKNOWLEDGMENTS}

We thanks Professor Shouhai Wang (Rice Research Institute, Anhui Academy of Agricultural Sciences) for supplying the original rice seeds of 'Guangzhan63S' and the ygl18 mutant.

\section{SUPPLEMENTARY MATERIAL}

The Supplementary Material for this article can be found online at: http://journal.frontiersin.org/article/10.3389/fpls.2017. 01694/full\#supplementary-material

Meinecke, L., Alawady, A., Schroda, M., Willows, R., Kobayashi, M. C., Niyogi, K. K., et al. (2010). Chlorophyll-deficient mutants of Chlamydomonas reinhardtii that accumulate magnesium protoporphyrin IX. Plant Mol. Biol. 72, 643-658. doi: 10.1007/s11103-010-9604-9

Mochizuki, N., Tanaka, R., Tanaka, A., Masuda, T., and Nagatani, A. (2008). The steady-state level of Mg-protoporphyrin IX is not a determinant of plastid-tonucleus signaling in Arabidopsis. Proc. Natl. Acad. Sci. U.S.A. 105, 15184-15189. doi: 10.1073/pnas.0803245105

Nagata, N., Tanaka, R., Satoh, S., and Tanaka, A. (2005). Identification of a vinyl reductase gene for chlorophyll synthesis in Arabidopsis thaliana and implications for the evolution of Prochlorococcus species. Plant Cell 17, 233-240. doi: 10.1105/tpc.104.027276

Pontier, D., Albrieux, C., Joyard, J., Lagrange, T., and Block, M. A. (2007). Knock-out of the magnesium protoporphyrin IX methyltransferase gene in Arabidopsis. J. Biol. Chem. 282, 2297-2304. doi: 10.1074/jbc.M610286200

Richter, A. S., Peter, E., Rothbart, M., Schlicke, H., Toivola, J., Rintamaki, E., et al. (2013). Post-translational influence of NADPH-dependent thioredoxin reductase $\mathrm{C}$ on enzymes in tetrapyrrole synthesis. Plant Physiol. 162, 63-73. doi: 10.1104/pp.113.217141

Richter, A. S., Wang, P., and Grimm, B. (2016). Arabidopsis Mg-Protoporphyrin IX Methyltransferase activity and redox regulation depend on conserved cysteines. Plant Cell Physiol. 57, 519-527. doi: 10.1093/pcp/pcw007

Sakuraba, Y., Rahman, M. L., Cho, S. H., Kim, Y. S., Koh, H. J., Yoo, S. C., et al. (2013). The rice faded green leaf locus encodes protochlorophyllide oxidoreductase B and is essential for chlorophyll synthesis under high light conditions. Plant J. 74, 122-133. doi: 10.1111/tpj.12110

Sheng, Z., Lv, Y., Li, W., Luo, R., Wei, X., Xie, L., et al. (2017). Yellow-Leaf 1 encodes a magnesium-protoporphyrin IX monomethyl ester cyclase, involved in chlorophyll biosynthesis in rice (Oryza sativa L.). PLoS ONE 12:e0177989. doi: 10.1371/journal.pone.0177989

Shepherd, M., and Hunter, C. N. (2004). Transient kinetics of the reaction catalysed by magnesium protoporphyrin IX methyltransferase. Biochem. J. 382(Pt 3), 1009-1013. doi: 10.1042/BJ20040661

Shepherd, M., Reid, J. D., and Hunter, C. N. (2003). Purification and kinetic characterization of the magnesium protoporphyrin IX methyltransferase from Synechocystis PCC6803. Biochem. J. 371(Pt 2), 351-360. doi: 10.1042/bj20021394

Strand, A., Asami, T., Alonso, J., Ecker, J. R., and Chory, J. (2003). Chloroplast to nucleus communication triggered by accumulation of $\mathrm{Mg}$-protoporphyrinIX. Nature 421, 79-83. doi: 10.1038/nature01204

Sun, C., Liu, L., Tang, J., Lin, A., Zhang, F., Fang, J., et al. (2011). RLIN1, encoding a putative coproporphyrinogen III oxidase, is involved in lesion initiation in rice. J. Genet. Genomics 38, 29-37. doi: 10.1016/j.jcg.2010.12.001

Ueda, M., Tanaka, A., Sugimoto, K., Shikanai, T., and Nishimura, Y. (2014). chlB requirement for chlorophyll biosynthesis under short photoperiod in Marchantia polymorpha L. Genome Biol. Evol. 6, 620-628. doi: 10.1093/gbe/evu045 
Vandesompele, J., De Preter, K., Pattyn, F., Poppe, B., Van Roy, N., De Paepe, A., et al. (2002). Accurate normalization of real-time quantitative RT-PCR data by geometric averaging of multiple internal control genes. Genome Biol. 3:RESEARCH0034. doi: 10.1186/gb-2002-3-7research0034

Van Wilder, V., De Brouwer, V., Loizeau, K., Gambonnet, B., Albrieux, C., Van Der Straeten, D., et al. (2009). C1 metabolism and chlorophyll synthesis: the Mg-protoporphyrin IX methyltransferase activity is dependent on the folate status. New Phytol. 182, 137-145. doi: 10.1111/j.1469-8137.2008. 02707.x

Wang, P., Gao, J., Wan, C., Zhang, F., Xu, Z., Huang, X., et al. (2010). Divinyl chlorophyll(ide) a can be converted to monovinyl chlorophyll(ide) a by a divinyl reductase in rice. Plant Physiol. 153, 994-1003. doi: $10.1104 /$ pp.110.158477

Wang, X., Huang, R., and Quan, R. (2017). Mutation in Mg-Protoporphyrin IX .monomethyl ester cyclase decreases photosynthesis capacity in rice. PLoS ONE 12:e0171118. doi: 10.1371/journal.pone.0171118

Wang, Z., Wang, Y., Hong, X., Hu, D., Liu, C., Yang, J., et al. (2015). Functional inactivation of UDP-N-acetylglucosamine pyrophosphorylase 1 (UAP1) induces early leaf senescence and defence responses in rice. J. Exp. Bot. 66, 973-987. doi: 10.1093/jxb/ eru456

Wang, Z., Wang, Y., Yang, J., Hu, K., An, B., Deng, X., et al. (2016). Reliable selection and holistic stability evaluation of reference genes for rice under 22 different experimental conditions. Appl. Biochem. Biotechnol. 179, 753-775. doi: 10.1007/s12010-016-2029-4
Wu, Z., Zhang, X., He, B., Diao, L., Sheng, S., Wang, J., et al. (2007). A chlorophylldeficient rice mutant with impaired chlorophyllide esterification in chlorophyll biosynthesis. Plant Physiol. 145, 29-40. doi: 10.1104/pp.107.100321

Yang, Y., Xu, J., Huang, L., Leng, Y., Dai, L., Rao, Y., et al. (2016). PGL, encoding chlorophyllide a oxygenase 1, impacts leaf senescence and indirectly affects grain yield and quality in rice. J. Exp. Bot. 67, 1297-1310. doi: 10.1093/jxb/erv529

Yu, C., Wang, L., Chen, C., He, C., Hu, J., Zhu, Y., et al. (2014). Protoplast: a more efficient system to study nucleo-cytoplasmic interactions. Biochem. Biophys. Res. Commun. 450, 1575-1580. doi: 10.1016/j.bbrc.2014.07.043

Zhang, H., Li, J., Yoo, J. H., Yoo, S. C., Cho, S. H., Koh, H. J., et al. (2006). Rice Chlorina-1 and Chlorina-9 encode ChlD and ChlI subunits of Mg-chelatase, a key enzyme for chlorophyll synthesis and chloroplast development. Plant Mol. Biol. 62, 325-337. doi: 10.1007/s11103-006-9024-z

Conflict of Interest Statement: The authors declare that the research was conducted in the absence of any commercial or financial relationships that could be construed as a potential conflict of interest.

Copyright (C) 2017 Wang, Hong, Hu, Wang, Wang, Du, Li, Hu, Cheng, An and Li. This is an open-access article distributed under the terms of the Creative Commons Attribution License (CC BY). The use, distribution or reproduction in other forums is permitted, provided the original author(s) or licensor are credited and that the original publication in this journal is cited, in accordance with accepted academic practice. No use, distribution or reproduction is permitted which does not comply with these terms. 